\title{
Calibration of Star-Formation Rate Measurements Across the Electromagnetic Spectrum
}

\author{
V. Buat ${ }^{1}$, J. Braine ${ }^{2}$, D. A. Dale ${ }^{3}$, A. Hornschemeier ${ }^{4}$, B. Lehmer $^{4,5}$, \\ P. Kroupa ${ }^{6}$ J. Pflamm-Altenburg ${ }^{6}$, C. C. Popescu ${ }^{7}, \mathbf{H} . \mathbf{W u}^{8}$ \\ and A. Zezas ${ }^{9}$
}

${ }^{1}$ Aix-Marseille Université, CNRS, LAM (Laboratoire d'Astrophysique de Marseille) UMR7326, 13388, France

email: veronique.buat@oamp.fr

${ }^{2}$ Observatoire de Bordeaux, Laboratoire d'Astrophysique de Bordeaux, 2 rue de l'Observatoire, BP 89, 33270 Floirac, France

email: Jonathan.Braine@obs.u-bordeaux1.fr

${ }^{3}$ Department of Physics \& Astronomy, University of Wyoming, Laramie, WY 82070 USA email: ddale@uwyo.edu

${ }^{4}$ NASA Goddard Space Flight Centre, Code 662, Greenbelt, MD 20771, USA

email: Ann.Hornschemeier@nasa.gov

${ }^{5}$ The Johns Hopkins University, Homewood Campus, Baltimore, MD 21218, USA email: blehmer@pha.jhu.edu

${ }^{6}$ Argelander-Institute for Astronomy, Auf dem Hügel 71, 53121 Bonn, Germany email: pavel, jpflamm@astro.uni-bonn.de

${ }^{7}$ Jeremiah Horrocks Institute, University of Central Lancashire, PR1 2HE, Preston, UK email: cpopescu@uclan.ac.uk

${ }^{8}$ Key Laboratory of Optical Astronomy, National Astronomical Observatories, Chinese Academy of Sciences, Beijing 100012, China

${ }^{9}$ Department of Physics, University of Crete, P.O. Box 2208, 71003 Heraklion, Crete, Greece

Institute of Electronic stucture and LASER, FORTH, Heraklion, Crete, Greece

Harvard-Smithnonoan Center for Astrophysics, 60 Garden Street, Cambridge, MA 02138, USA

email: azezas@physics.uoc.gr

\begin{abstract}
Star-formation is one of the main processes that shape galaxies, and together with black-hole accretion activity the two agents of energy production in galaxies. It is important on a range of scales from star clusters/OB associations to galaxy-wide and even group/cluster scales. Recently, studies of star-formation in sub-galactic and galaxy-wide scales have met significant advances owing to: (a) developments in the theory of stellar evolution, stellar atmospheres, and radiative transfer in the interstellar medium; (b) the availability of more sensitive and higher resolution data; and (c) observations in previously poorly charted wavebands (e.g. Ultraviolet, Infrared, and X-rays). These data allow us to study more galaxies at ever-increasing distances and nearby galaxies in greater detail, and different modes of star formation activity such as massive star formation and low level continuous star formation in a variety of environments. In this contribution we summarize recent results in the fields of multi-wavelength calibrations of star-formation rate indicators, the Stellar Initial Mass function, and radiative transfer and modeling of the Spectrale Energy Disrtributions of galaxies.
\end{abstract}

Keywords. stars: luminosity function, mass function, binaries (including multiple): close, galaxies: star clusters, galaxies: fundamental parameters, infrared: galaxies, ultraviolet: galaxies, X-rays: binaries 


\section{Multi-wavelength Calibrations of SFR}

\subsection{General Characteristics of Star Formation Calibrations}

Measuring accurate star formation rates (SFRs) is a crucial step in any study of galaxy evolution. As an example, the evolution of the SFR density with redshift is one of the major diagnostics to study galaxy evolution and any model of galaxy formation and evolution must reproduce this evolution. Before drawing any firm conclusions about the validity of theoretical models it must be stressed that large uncertainties come from the difficulty in measuring accurately the star formation rate (e.g., Davé 2008). Various calibrators are used, covering the full electromagnetic spectrum, from the X-ray through the ultraviolet (UV), the optical, the infrared (IR), all the way to the radio. Both continuum emission and emission lines have been calibrated in terms of SFR. The derivation of SFR relies on conversion factors depending on the indicator used. In this subsection we present the general way to derive SFR from direct stellar emission and then discuss the uncertainties of the calibrations due to variations of the star formation histories. The effect of dust attenuation will be discussed in Section 1.3. Complete reviews of different star formation indicators were published recently (Calzetti 2012; Kennicutt \& Evans 2012; Boissier 2012).

\subsubsection{Basic Equation and Standard Calibrations}

The fundamental equation to link the intrinsic luminosity emitted by stars at wavelength $\lambda$ and time $t$ is

$$
L(\lambda, t)=\int_{0}^{t} \int_{M_{l o w}}^{M_{u p}} F_{\lambda}(m, \theta) \operatorname{SFR}(t-\theta) \psi(m) \mathrm{d} m \mathrm{~d} \theta
$$

where $F_{\lambda}(m, \theta)$ are the evolutionary stellar tracks, $\psi(m)$ the initial mass function, and $S F R(t)$ the star formation rate. From this fundamental equation there are two ways to

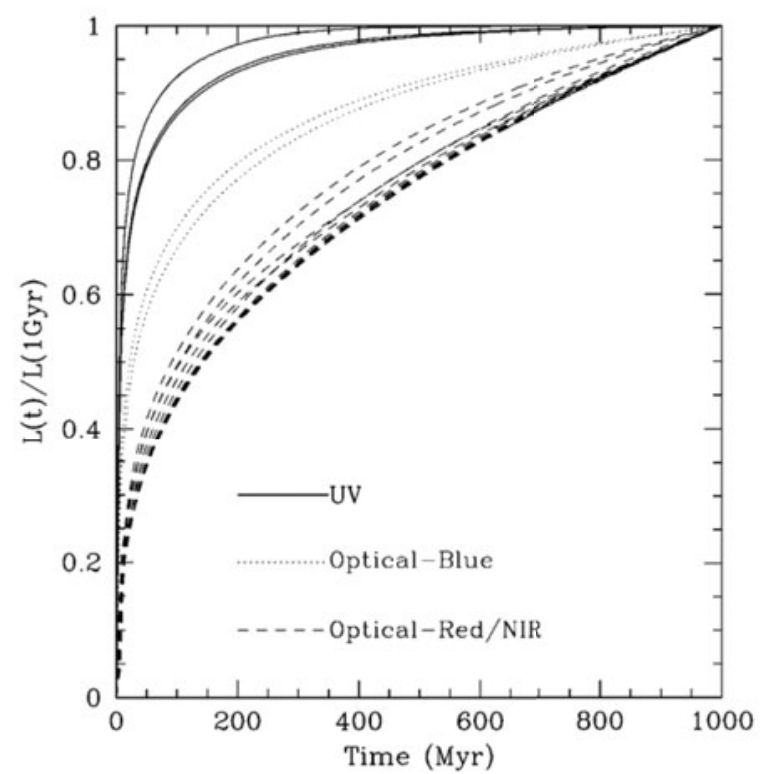

Figure 1. Evolution of luminosity for several filters from the UV to the NIR for a constant $\mathrm{SFR}$ of $1 \mathrm{M}_{\odot} \mathrm{yr}^{-1}$. The luminosity is normalised to its value obtained after 1 Gyr. See Boissier (2012) for more details. 
derive SFRs. One can use stellar population synthesis models to fit a large set of data at different wavelengths; the current SFR is an output parameter of the fit (with the stellar mass and other parameters depending on the specific code used). Another popular way to proceed is to derive simple recipes. One assumes a SFR constant over a timescale $\tau$ and the SFR becomes simply proportional to the luminosity integrated over $\tau$. The timescale $\tau$ is chosen in order that the luminosity at wavelength $\lambda$ reaches a steady state:

$$
S F R=\left(\int_{0}^{t} \int_{M_{l o w}}^{M_{u p}} F_{\lambda}(m, \theta) S F R(t-\theta) \psi(m) \mathrm{d} m \mathrm{~d} \theta\right)^{-1} \times L(\lambda)
$$

The value of the conversion factor $C=S F R / L(\lambda)$ is calculated with a spectral synthesis code. The SFR calculated using a conversion factor can be different from the average of the star formation activity during $\tau$ if the actual SFR is strongly varying during this period. The assumption of a constant SFR is likely to be valid on short timescales only and SFR should be derived from the emission of stars with short lifetimes, preferentially in the ultraviolet or for recombination lines of ionizing photons. The evolution of the luminosity at different wavelength is illustrated in Figure 1. It is clearly seen that the timescale to reach a steady state increases with wavelength from $\sim 100$ Myr in the ultraviolet to more than 1 Gyr in optical-near IR. The timescale found for ionizing photons (i.e., recombination lines) is of the order of few Myr (Boissier 2012; Kennicutt \& Evans 2012). There is some evidence (see next subsection) for a SFR constant over a few hundred Myr, at least in the nearby universe; standard calibrations can be calculated, as was done in the popular review of Kennicutt (1998). The calibration depends also strongly on the initial mass function (e.g., Meurer et al. 2009, Pflamm-Altenburg et al. 2009) as discussed in Section 3 of this review.

\subsubsection{Impact of a Varying Star Formation Rate}

The assumption of a constant star formation rate is quite strong. It is important to check that the condition is fulfilled before using standard calibrations. In the nearby universe the tight correlation found between the $\mathrm{H} \alpha$ and UV luminosity of galaxies is a strong argument in favor of a rather constant star formation rate for whole galaxies over
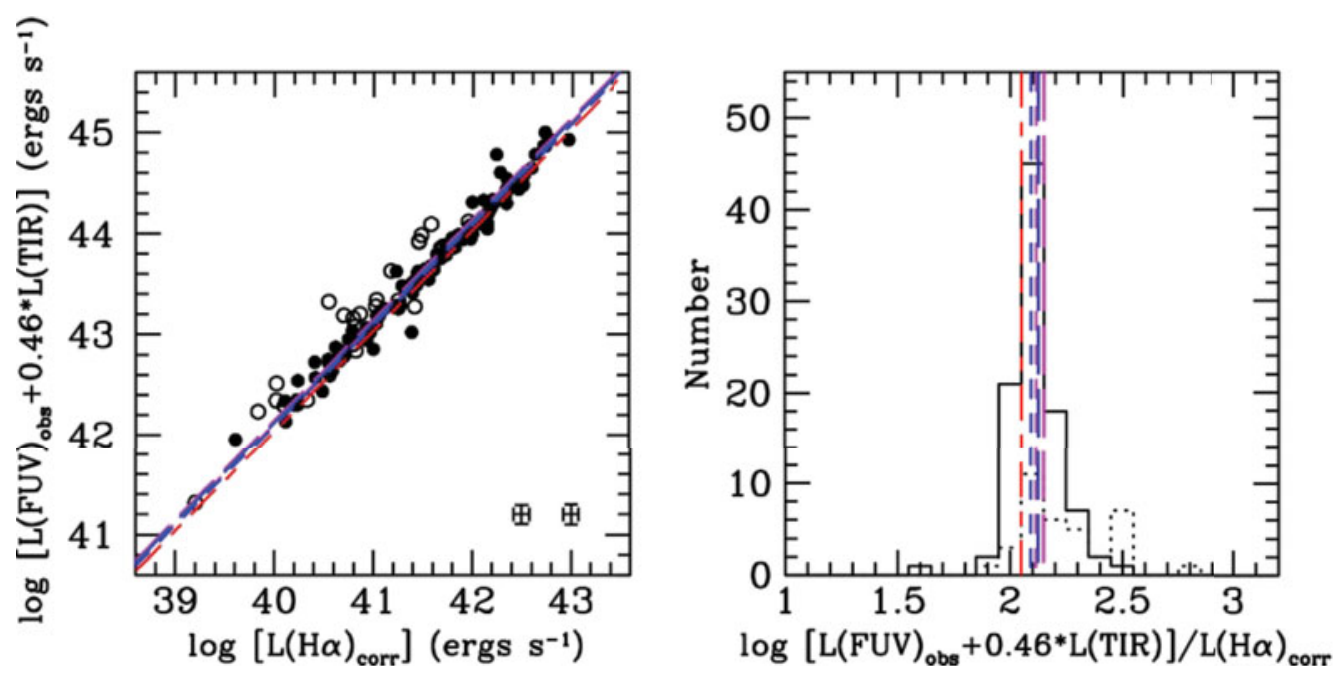

Figure 2. UV luminosities corrected for dust attenuation plotted against the $\mathrm{H} \alpha$ ones for galaxies in the nearby universe; both luminosities are corrected for dust attenuation. See Hao et al. (2011) for more details. 
the typical timescale for the UV light to reach a steady state (i.e., $\sim 300 \mathrm{Myr}$ ) as illustrated in Figure 2. Nearby dwarf galaxies are likely to experience rapid changes in their star formation rates (Weisz et al. 2012), implying an apparent deficit of $\mathrm{H} \alpha$ emission with respect to the UV one. However, it was found difficult to distinguish between variations of the recent star formation history and that of the initial mass function by comparing the $\mathrm{H} \alpha$ and UV emission of these objects (Meurer et al. 2009; Boselli et al. 2009; Lee et al. 2009; Fumagalli et al. 2011; Weiz et al. 2012).

At high redshift, timescales become shorter and the assumption of a constant star formation rate over several hundreds of Myr may not be valid. It is nicely illustrated by Schaerer et al. (2013) who explored a large number of star formation histories described by declining or rising functions in addition to constant and delayed star formation. Their models and the corresponding ratio of the SFR to the bolometric stellar luminosity $L_{\mathrm{bol}}$ is shown in Figure 3. At $z \simeq 4$ Schaerer et al. show that the standard calibrations assuming constant star formation must be used with much caution, with strong implications on the SFR-stellar mass relation.

\subsection{Far-Infrared Calibrations}

In the IRAS and ISO eras (e.g., Hunter et al. 1986; Lehnert \& Heckman 1996), infrared luminosities from wavelengths less than $200 \mu \mathrm{m}$ proved to be good star formation rate tracers. Since the successful launch of the Spitzer Space Telescope, infrared luminosities have been widely used as important tools to calculate the star formation rates of galaxies. Recent studies enabled by Spitzer observations indicate that mid-IR luminosities (such as $8 \mu \mathrm{m}$ and $24 \mu \mathrm{m}$ ) of galaxies also have good correlations with $1.4 \mathrm{GHz}$ and $\mathrm{H} \alpha / \mathrm{P} \alpha$ luminosities (e.g. Wu et al. 2005; Calzetti et al. 2007; Zhu et al. 2008; Kennicutt et al. 2009; Calzetti et al. 2010).

However, Alonso-Herrero et al. (2006) shows that the Spitzer- $8 \mu \mathrm{m}-\mathrm{P} \alpha$ relations are different for nuclear regions and HII regions of luminous infrared galaxies, possibly because of diffuse $8 \mu \mathrm{m}$ emission associated with nuclear regions. Also many dwarf galaxies do not follow the general correlation of star-forming galaxies (Wu et al. 2005). What
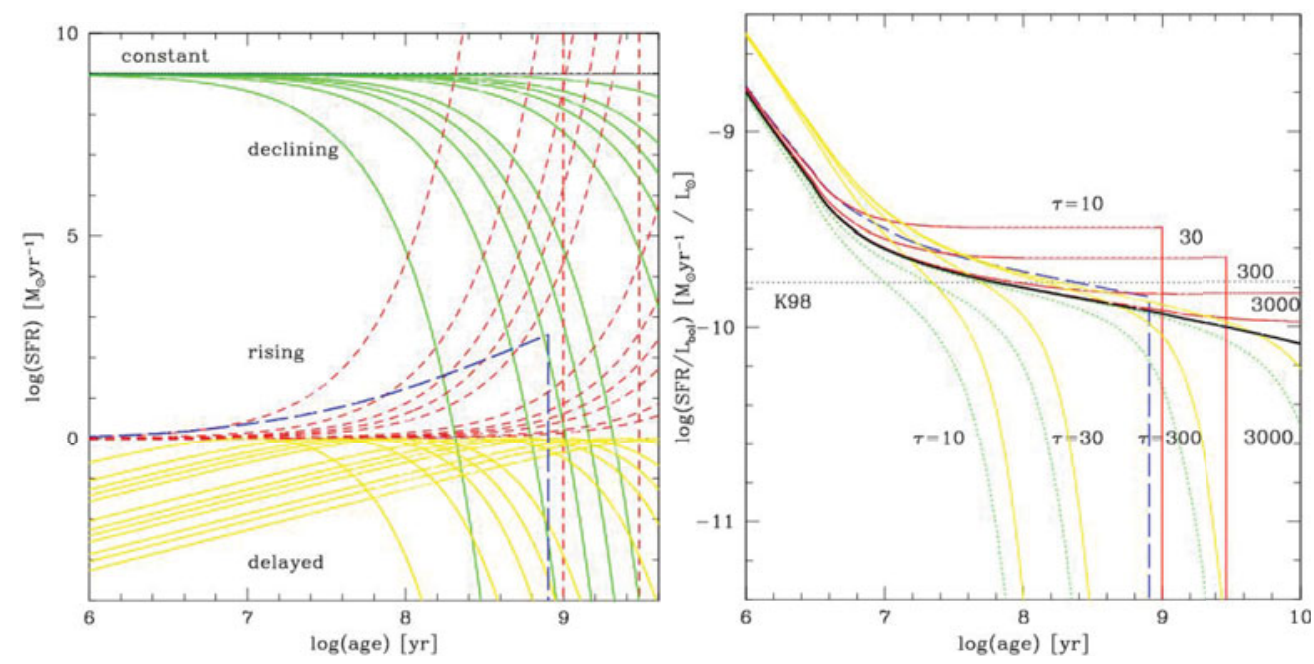

Figure 3. From Schaerer et al. (2012). Left panel: various star formation histories. Right panel: $S F R / L_{\mathrm{bol}}$ for the various star formation histories as a function of time. 
would affect the mid-IR properties? Many efforts (Engelbracht et al. 2005; Wu et al. 2006; Wu et al. 2007; Siebenmorgen et al. 2004) have shown that both metallicity and AGN activity can affect the mid-IR properties of galaxies. This will bias the estimate of star formation rates. Some other factors, such as extinction and morphological type (Li et al. 2007) can also affect the calculation of star formation rates. In fact the most intrinsic factors involve the radiation field and dust. The strength and intensity of the radiation field, as well as the chemical content and the distribution of dust grain sizes, determine the deviation of the mid-IR-SFR relation for star-forming galaxies. Meanwhile, in many early-type galaxies, the contamination from the some diffuse regions, which are associated with old stellar populations rather than with current star formation, can also play important roles in the emission arising from mid-IR bands.

As the mid-IR luminosity can trace the dust-obscured star formation and the $\mathrm{H} \alpha$ luminosity can trace the unobscured star formation, a combined $\mathrm{H} \alpha / 24 \mu \mathrm{m}$ luminosity is introduced by Calzetti et al. (2007) and can trace the star formation rates almost linearly (see also $\S 1.3$ ). Zhu et al. (2008) and Kennicutt et al. (2009) confirmed Calzetti et al. (2007)'s results and obtain the similar relation for combined $\mathrm{H} \alpha / 8 \mu \mathrm{m}$ luminosity. The combined $\mathrm{H} \alpha /$ mid-IR luminosities are more robust to flucgtuations in radiation field and dust content, and thus give an overall more reliable estimate of galaxy SFRs. For example, dwarf galaxies obey the same correlation as normal star-forming galaxies. However, as Spitzer was not a survey facility and its coverage was limited to a relatively small portion of the sky, the mid-IR bands cannot be widely used as star formation rate indicators. After the release of the AKARI full sky survey (Yuan et al. 2011), the mid-IR luminosity could be widely employed. Moreover, the WISE $22 \mu \mathrm{m}$ band is quite similar to the Spitzer $24 \mu \mathrm{m}$ band.

After cross-matching star-forming galaxy data between WISE and that of SDSS, a similar correlation was established between the combined $\mathrm{H} \alpha / 22 \mu \mathrm{m}$ luminosity and extinction-corrected $\mathrm{H} \alpha$ luminosity (Figure 4) as Zhu et al. (2008) did for Spitzer $24 \mu \mathrm{m}$. The coefficient $b=0.020$ is similar to that of Zhu et al. (2008) and Kennicutt et al. (2009). For galaxies at higher redshift $(z>0.35)$ with only [OII]3727 emission line data available, the [OII] 3727 luminosity was employed instead of the $\mathrm{H} \alpha$ luminosity; a good correlation between the combined [OII] $3727 / 22 \mu \mathrm{m}$ luminosity and extinction-corrected $\mathrm{H} \alpha$ luminosity is shown in Figure 4.

At longer (sub-millimeter) wavelengths the properties of galaxies were still unclear before the Herschel era. The Herschel data opened a new window to studying the cool dust universe. For example, Dominguez et al. (2012) found the total IR luminosity is a good star formation tracer in nearby galaxies by using Herschel PACS data. However, whether or not a single SPIRE sub-millimeter band of Herschel can trace star formation of galaxies is still questionable. Using Herschel ATLAS science demonstration phase data crossidentified with SDSS DR7 spectra, a sample of 297 galaxies was constructed and classified into five morphological types; more than $40 \%$ of galaxies are peculiar/compact galaxies. The peculiar galaxies show higher far-infrared/sub-millimeter luminosity-to-mass ratios than other types. Wu and collaborators analyzed the correlations of far-infrared/submillimeter and $\mathrm{H} \alpha$ luminosities for different morphological types and different spectral types. The Spearman rank coefficient decreases and the scatter increases with the wavelength increasing from $100 \mu \mathrm{m}$ to $500 \mu \mathrm{m}$. These trends indicate that a single Herschel SPIRE band is not adequate for tracing star formation activity in galaxies. Finally, AGNs contribute proportionally less to the far-infrared/sub-millimeter luminosity; AGN galaxies show little difference from star-forming galaxies in these diagnostics. However, the earlier type galaxies present significant deviations from the best-fitting of star-forming galaxies. 

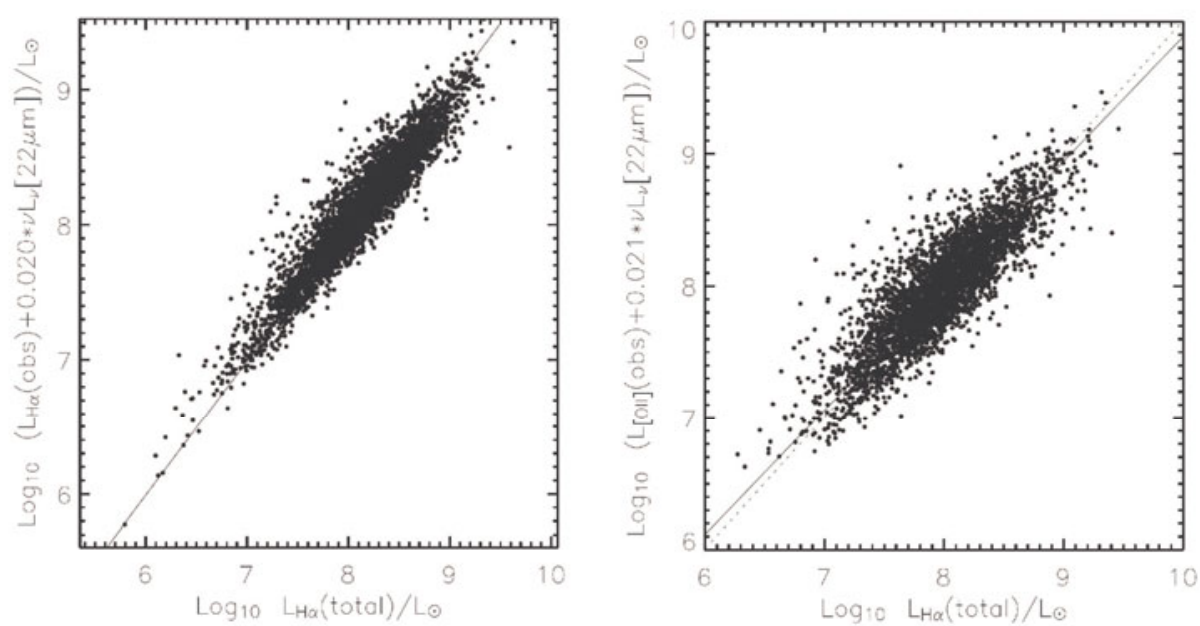

Figure 4. Correlations between the combined $\mathrm{H} \alpha / W I S E(22 \mu \mathrm{m})$ luminosity, the combined [OII] $3727 / W I S E(22 \mu \mathrm{m})$ luminosity, and the extinction-corrected $\mathrm{H} \alpha$ luminosity.

\subsection{Composite Indicators of Star Formation Rates}

Composite star formation rate indicators combine emission from unobscured star formation at wavelength $\lambda_{1}$ and emission from obscured star formation that reappears at wavelength $\lambda_{2}$,

$$
L\left(\lambda_{1}\right)_{\text {corrected }}=L\left(\lambda_{1}\right)_{\text {observed }}+a L\left(\lambda_{2}\right)
$$

where $L\left(\lambda_{1}\right)_{\text {corr }}$ has been previously calibrated as a star formation rate indicator (e.g., Kennicutt 1998; see also Figure 2). The ability to reliably combine two photometric measurements to yield an extinction-corrected SFR is a powerful tool for situations where spectroscopy-based extinction estimates are unavailable. Recent examples of such combinations include $\mathrm{H} \alpha+24 \mu \mathrm{m}$ or IR+UV (Hirashita et al. 2003; Iglesias-Páramo et al. 2006; Buat et al. 2007; Calzetti et al. 2007; Elbaz et al. 2007; Daddi et al. 2007; Leroy et al. 2008; Zhu et al. 2008; Kennicutt et al. 2009; Liu et al. 2011; Wuyts et al. 2011). A direct corollary to this approach is that the ratio of the obscured and unobscured components can be used to estimate the amount of attenuation by dust (Prescott et al. 2007; Moore et al. 2010; Hao et al. 2011), i.e.,

$$
\begin{aligned}
& A(\mathrm{H} \alpha)=2.5 \log \left[1+a(\mathrm{H} \alpha) \nu L_{\nu}(24 \mu \mathrm{m}) / L(\mathrm{H} \alpha)\right] \\
& A(\mathrm{FUV})=2.5 \log [1+a(\mathrm{FUV}) L(\mathrm{IR}) / L(\mathrm{FUV})]
\end{aligned}
$$

The latter relation involves the so-called "infrared excess" that is frequently used to quantify the amount of attenuation by dust in galaxies (e.g., Meurer et al. 1995; see Figure 5).

The dependence of the calibration constant $(a(\mathrm{H} \alpha)$ or $a(\mathrm{FUV})$ in the above two instances) on region size is described in $\S 1.4$. Table 1 summarizes these calibration coefficients from literature studies of either "global" (spatially-integrated) emission or localized emission, where available.

The scatter for all the different (global) composite indicators are similarly (and somewhat surprisingly) tight. In contrast, corrections for UV extinction via UV colors result in nonlinearities and scatters 2.5 times larger (Hao et al. 2011). 
Table 1. Coefficients for Select Composite SFR Tracers

\begin{tabular}{llcll}
\hline Composite SFR Relation & Coefficient $a$ & $\begin{array}{c}\text { Dispersion } \\
(\mathrm{dex})\end{array}$ & Region & Reference \\
\hline$L(\mathrm{H} \alpha)_{\text {corr }}=L(\mathrm{H} \alpha)_{\text {obs }}+a L(8 \mu \mathrm{m})$ & $0.011 \pm 0.003$ & 0.11 & Global & $(1),(2)$ \\
$L(\mathrm{H} \alpha)_{\text {corr }}=L(\mathrm{H} \alpha)_{\text {obs }}+a L(24 \mu \mathrm{m})$ & $0.020 \pm 0.005$ & 0.12 & Global & $(1),(2)$ \\
$L(\mathrm{H} \alpha)_{\text {corr }}=L(\mathrm{H} \alpha)_{\text {obs }}+a L(24 \mu \mathrm{m})$ & $0.031 \pm 0.006$ & 0.3 & Local & $(3)$ \\
$L(\mathrm{H} \alpha)_{\text {corr }}=L(\mathrm{H} \alpha)_{\text {obs }}+a L(70 \mu \mathrm{m})$ & $0.011 \pm 0.001$ & 0.24 & Local & $(4)$ \\
$L(\mathrm{H} \alpha)_{\text {corr }}=L(\mathrm{H} \alpha)_{\text {obs }}+a L(\mathrm{TIR})$ & $0.0024 \pm 0.0006$ & 0.09 & Global & $(1)$ \\
$L(\mathrm{FUV})_{\text {corr }}=L(\mathrm{FUV})_{\text {obs }}+a L(\mathrm{TIR})$ & $0.46 \pm 0.12$ & 0.09 & Global & $(5)$ \\
$L(\mathrm{FUV})_{\text {corr }}=L(\mathrm{FUV})_{\text {obs }}+a L(25 \mu \mathrm{m})$ & $3.89 \pm 0.15$ & 0.13 & Global & $(5)$ \\
\hline \hline
\end{tabular}

References: (1) Kennicutt et al. 2009; (2) Zhu et al. 2008; (3) Calzetti et al. 2007; (4) Li et al. 2013; (5) Hao et al. 2011

\subsection{SFR Calibrations and Their Dependence on Region Size}

Inspection of Table 1 shows a $\sim 35 \%$ difference in the coefficient between the global and local $\mathrm{H} \alpha+24$ SFR indicators. The reason for this discrepancy is tied to the sizescale over which the measurements are carried out. While the global coefficient stems from studies of spatially-integrated emission, the local coefficient derives from the Calzetti et al. (2007) study of "HII knots" of size 200-600 pc. An important aspect of the Calzetti et al. study is the subtraction of the "background" emission from each local photometric measurement, which effectively removes the smooth galaxy emission that underlies each HII knot. Comparisons with models shows that perhaps up to $50 \%$ of a galaxy's infrared emission derives from interstellar dust grains heated by the diffuse radiation field that is unrelated to localized sites of star formation (Kennicutt et al. 2009).

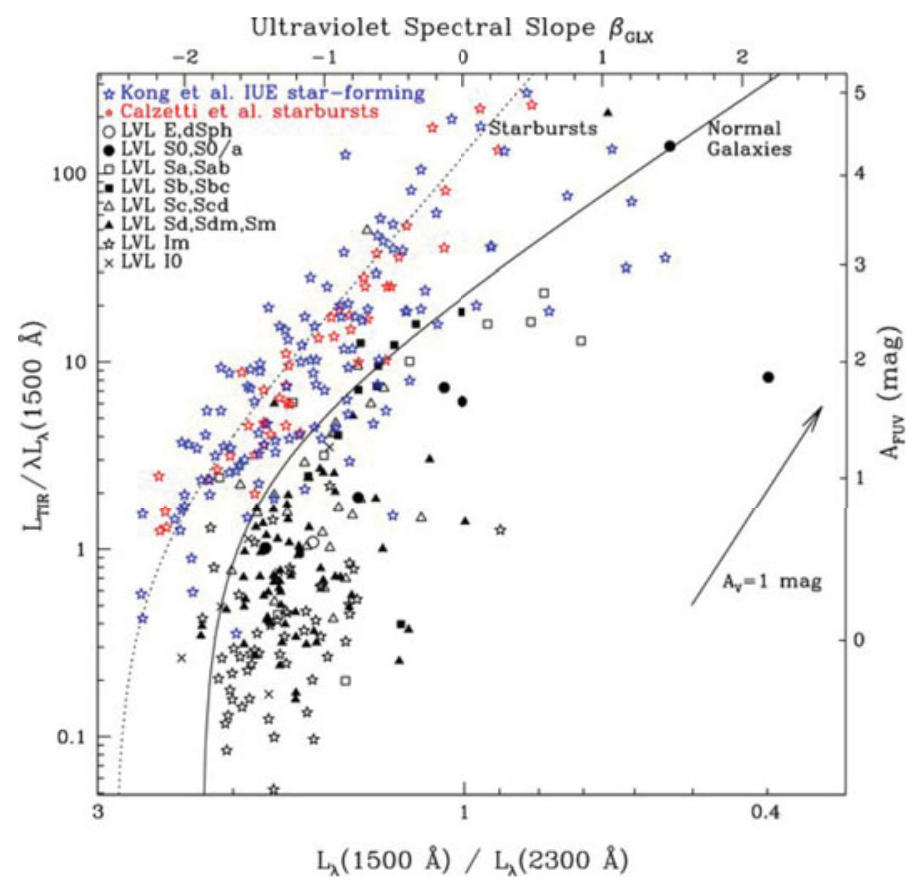

Figure 5. Example of the "IRX- $\beta$ " relation for quantifying attenuation by dust as a function of observed UV color (from Dale et al. 2009). The relation is tighter for starbursting systems, and more loosely defined for normal star-forming galaxies. 
Likewise, infrared monochromatic SFR indicators can also show a region size dependence, and for similar reasons as described above. For example, Li et al. (2013) find that the SFR calibration coefficient for $70 \mu \mathrm{m}$ data changes by a factor $\sim 2$ for apertures spanning $0.1-10 \mathrm{kpc}$; their interpretation is that $\sim 50 \%$ of the total $70 \mu \mathrm{m}$ emission comes from dust that is heated by stellar populations not associated with current star formation (see Figure 6).

\subsection{Measuring star formation rates with Spectral Energy Distributions}

Star formation rates are basic output parameters of codes aimed at fitting spectral energy distributions (SED). By comparing data with models, one can attempt to derive some physical parameters related to the star formation history and dust attenuation in a homogeneous way and simultaneously for all galaxies of a given sample and at different redshifts. In practice, the current SFR, the stellar mass, and the amount of dust attenuation are commonly derived from broad-band photometry. Without spectral information and given the high degree of degeneracy in the SEDs, details about the star formation history are difficult to constrain implying an uncertainty on the determination of SFR. We refer to Walcher et al. (2010) for an exhaustive review on SED fitting techniques.

With the availability of mid- and far-IR data for large samples of galaxies, codes that combine stellar and dust emission to analyse SEDs are particularly useful. The standard approach consists of solving the radiation transfer in model galaxies to build self-consistent SEDs from the UV to the far-IR (see e.g. Bruzual \& Charlot, 2003, for a detailed description of these models, or refer to Popescu et al. 2011, and Silva et al. 1998). These sophisticated models require complex calculations and are not directly applicable to large samples of galaxies. Another tool consists of models based on a simple energetic budget where the global dust emission corresponds to the difference between the emitted and observed stellar light (da Cunha et al. 2008; Noll et al. 2009). These codes, based on synthetic stellar population modeling and simple emission properties for the dust, are well suited to analysing large datasets at the expense of an oversimplification of the physical processes at work in galaxies. An example of spectral energy distributions generated by the CIGALE code (Noll et al. 2009; http://cigale.oamp.fr) is shown in Figure7.

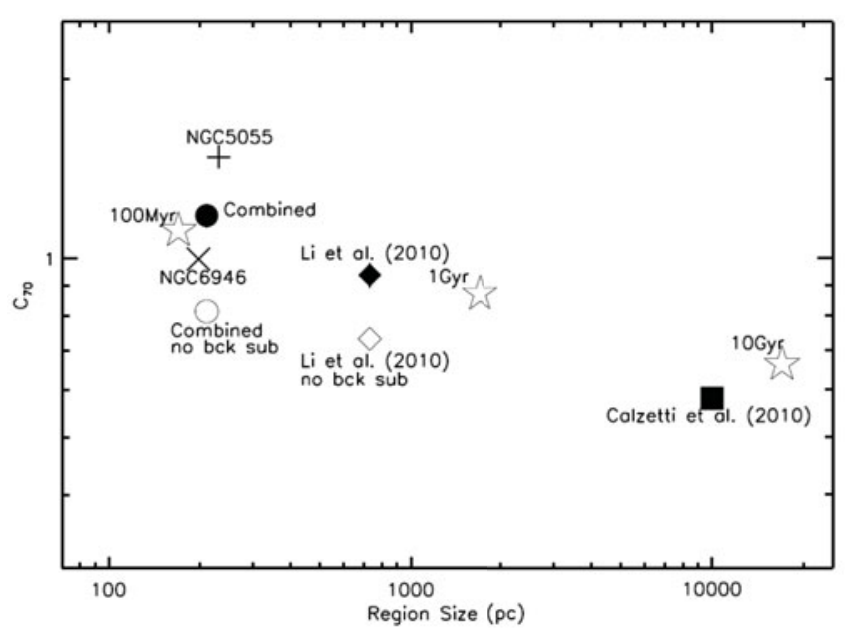

Figure 6. Region size dependence of the $70 \mu \mathrm{m}$ SFR calibration coefficient (taken from Li et al. 2013). 


\subsection{X-ray Emission as a Star-Formation Rate Tracer}

X-ray emission from normal galaxies (i.e., not dominated by luminous active galactic nuclei $[\mathrm{AGN}]$ ) originates from a variety of components that vary dependent upon galaxy type and physical properties (see, e.g., Fabbiano 1989, 2006 for reviews). For late-type star-forming galaxies, X-ray emitting populations associated with both young and old stellar populations are expected to provide dominant contributions (i.e., high-mass and low-mass X-ray binaries, supernovae and their remnants, hot gas from starburst flows, and young stars). Passive early-type galaxies, without significant star-formation activity, have X-ray emission dominated by low-mass X-ray binaries (LMXBs) and hot X-ray emitting gas (e.g., O’Sullivan et al. 2001; Gilfanov 2004; Boroson et al. 2011). For starforming galaxies, it has been observed that the X-ray power output is directly correlated with the emission from other wavebands that are associated with star-formation activity (e.g., UV, $\mathrm{H} \alpha$, total IR, and radio), suggesting that X-ray emission may provide a useful measure of the current SFR in galaxies (hereafter the X-ray/SFR correlation). Such a prospect is attractive from a practical standpoint, since X-ray emission is penetrating in nature, and therefore has the potential to provide an independent view of star-formation activity in galaxies that does not suffer significantly from absorption. Understanding the calibration of the X-ray/SFR correlation, its scatter, and its cosmic evolution has been a rapidly advancing area of research. In this section we briefly highlight some recent results with emphasis on hard X-ray emission as a tracer of the SFR.

\subsubsection{The X-ray/SFR Correlation in the Local Universe}

The local X-ray/SFR correlation has been calibrated for star-forming galaxies over $\sim 5$ orders of magnitude in SFR (e.g., Ranalli et al. 2003; Colbert et al. 2004; Persic \& Rephaeli 2007; Lehmer et al. 2010; Iwasawa et al. 2009; Pereira-Santaella et al. 2011; Mineo et al. 2012a,b). Given the multiple components contributing to the X-ray emis-

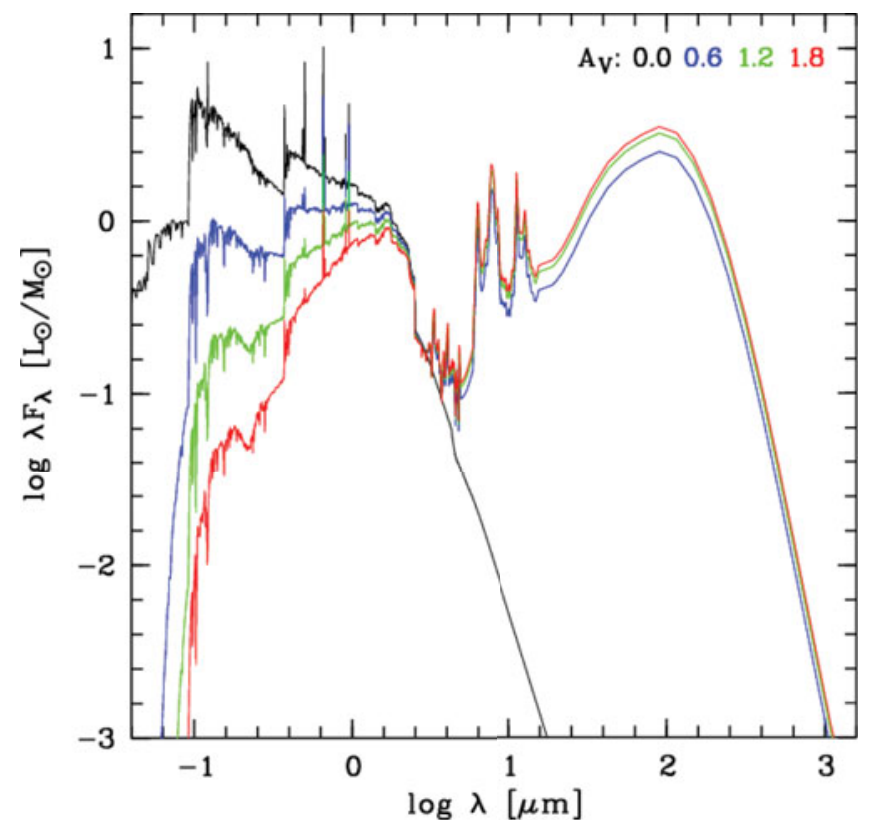

Figure 7. Example of SEDs generated by the CIGALE code assuming an age of 1 Gyr, an exponentially decreasing SFR with a decay time $\tau=10$ Gyr and different dust attenuation in the $V$ band (from Noll et al. 2009). 
sion from normal galaxies, recent calibrations of the X-ray/SFR correlation have focused on separating these components either spectrally (e.g., Lehmer et al. 2010 make use of $2-10 \mathrm{keV}$ emission dominated by X-ray binaries), or by spatially decomposing the emitting components into point sources (X-ray binaries) and diffuse emission (hot gas, unresolved binaries, and stars; see, e.g., Mineo et al. 2012a,b). Thanks to resolved imaging of nearby galaxies with Chandra and XMM-Newton, we know that the X-ray/SFR correlation at energies above $\sim 1 \mathrm{keV}$ is primarily driven by bright $\mathrm{X}$-ray binaries. Of the bright X-ray binaries in star-forming galaxies, LMXBs and HMXBs are expected to provide substantial contributions, which are expected to trace the relatively old and young stellar populations, respectively.

To account for the LMXB and HMXB contributions to the galaxy-wide hard X-ray emission, Lehmer et al. (2010) gathered a Chandra-observed sample of 66 nearby normal galaxies with good measurements of SFR and $M_{\star}$ and fit their data to the following relation:

$$
L_{2-10 \mathrm{keV}}=\alpha M_{\star}+\beta \mathrm{SFR}
$$

where $L_{2-10 \mathrm{keV}}$ is the galaxy-wide integrated $2-10 \mathrm{keV}$ power output, and $\alpha$ and $\beta$ are constants, which to first order account for the emission of LMXBs and HMXBs, and their scaling with $M_{\star}$ and SFR, respectively. For a Kroupa (2001) IMF, the best-fit values of these constants are $\alpha=(9.05 \pm 0.37) \times 10^{28} \operatorname{ergs~s}^{-1} M_{\odot}^{-1}$ and $\beta=(1.62 \pm$ $0.22) \times 10^{39} \mathrm{ergs} \mathrm{s}^{-1}\left(M_{\odot} \mathrm{yr}^{-1}\right)^{-1}$. The residual scatter in this relation is $\approx 0.34 \mathrm{dex}$, which is a $\approx 0.14$ dex improvement over a direct scaling of a direct linear scaling of $L_{2-10 \mathrm{keV}}$ vs. SFR. More recently, Mineo et al. (2012a) studied in detail nearby galaxies with high specific-SFRs (SFR/ $M_{\star}>10^{-10} \mathrm{yr}^{-1}$ ) and utilized spatial decomposition of X-ray point sources to directly compute the HMXB X-ray luminosity functions of each galaxy. Through this independent analysis, they provided a direct calibration for how the HMXB luminosity of galaxies scales linearly with SFR (i.e., $\beta$ from Equation 1.6). This analysis produced a scaling similar to that found by Lehmer et al. (2010) once differences between X-ray bandpass and IMF were accounted for. Despite the general agreement between the X-ray/SFR correlation, there remains significant scatter on the order of $\approx 0.3-0.4$ dex. The remaining scatter is likely to be both intrinsically statistical and physical in nature. Some unaccounted for physical dependencies to the correlation in Equation 1.6 include dependencies on stellar age and metallicity (see, e.g., the models from Fragos et al. 2013).

\subsubsection{Cosmic Evolution of the X-ray/SFR Correlation}

With the advent of ultradeep surveys with Chandra (e.g., the Chandra Deep Fields; see Brandt \& Hasinger 2005 and Brandt \& Alexander 2010 for reviews), it has become possible to study X-ray faint, cosmologically-distant normal galaxies, the majority of which are powered by star-formation processes. At the flux limit of the deepest X-ray survey to date, the 4 Ms Chandra Deep Field-South (CDF-S; Xue et al. 2011), the sky density of normal galaxies may already be larger than that of AGN (Lehmer et al. 2012). Initial investigations have placed first-order constraints on the evolution of the normalgalaxy X-ray luminosity function (e.g., Norman et al. 2004; Ptak et al. 2007; Tzanavaris \& Georgantopolous 2008), which have provided evidence for a rapidly increasing normalgalaxy X-ray luminosity density with redshift (proportional to $[1+z]^{2-4}$ ), consistent with the rising star-formation rate density of the Universe (e.g., Hopkins et al. 2004).

Analysis of the X-ray detected normal galaxies have shown that the X-ray/SFR correlation appears to hold out to $z \approx 1$ (e.g., Cowie et al. 2012; Mineo et al. 2012c; Ranalli et al. 2012; Symeonidis et al. 2012; Vattakunnel et al. 2012). Powerful X-ray stacking 

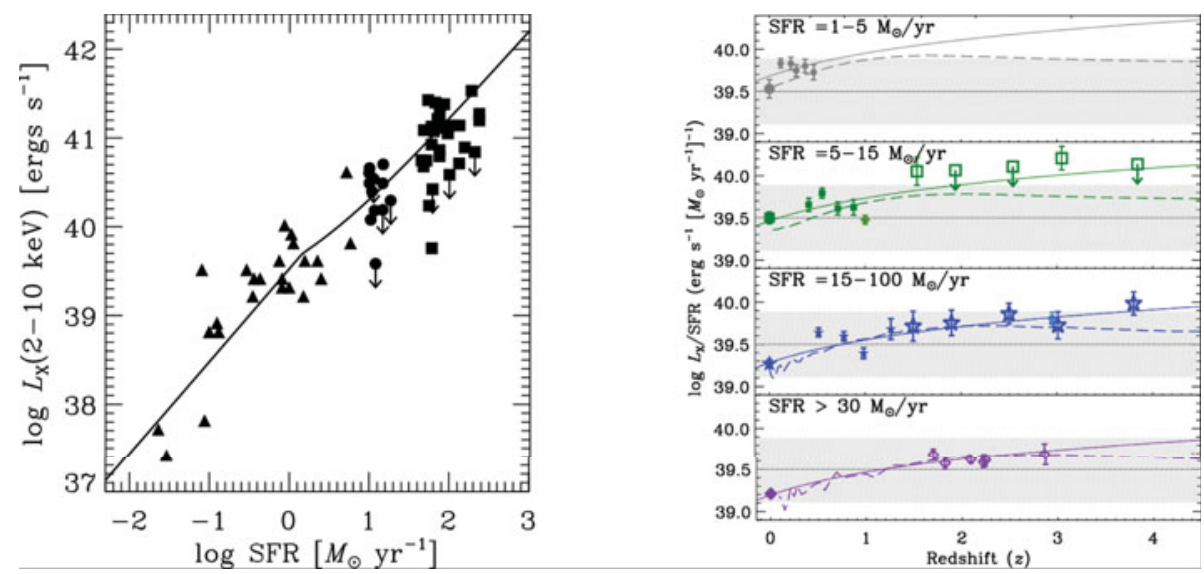

Figure 8. (Left Panel) The local X-ray/SFR correlation from Lehmer et al. (2010). The data shown in this plot are compiled from local normal galaxies (triangles; Colbert et al. 2004), LIRGs (circles; Lehmer et al. 2010), and LIRGs/ULIRGs (black squares; Iwasawa et al. 2009). The solid line represents the expected correlation given how HMXB and LMXB emission scale with SFR and $M_{\star}$, respectively, and how $M_{\star}$ scales with SFR. (Right Panel) Mean values of $L_{2-10 \mathrm{keV}} / \mathrm{SFR}$ from the stacking analyses presented in Basu-Zych et al. (2012). The best-fit redshift-dependent parameterization is shown as solid curves (Equation 1.6.2), and the population synthesis prediction from Fragos et al. (2013) is shown with dashed curves.

studies of large populations of normal star-forming galaxies selected by morphology and galaxy physical properties (e.g., SFR and stellar mass) have provided more sensitive insight into how the X-ray/SFR correlation has evolved over the majority of cosmic history. Using the 4 Ms CDF-S, Basu-Zych et al. (2012) utilized results from the literature (e.g., Reddy \& Steidel 2004; Laird et al. 2006; Lehmer et al. 2008) and large samples of thousands of Lyman-break galaxies to show that the X-ray/SFR correlation undergoes very mild evolution out to $z \approx 4$, which can be parameterized as follows:

$$
\log L_{2-10 \mathrm{keV}}=A \log (1+z)+B \log \mathrm{SFR}+C,
$$

where $A=0.93 \pm 0.07, B=0.65 \pm 0.03$, and $C=39.80 \pm 0.03$ are fitting constants. Additional indirect support for mild evolution of the X-ray/SFR correlation with redshift has been provided by Dijkstra et al. (2012) who used the observed SFR density evolution of the Universe to show that the unresolved SB CXRB can be fully explained by a $L_{\mathrm{X}} / \mathrm{SFR}$ ratio $\propto(1+z)^{b}$, where $b$ is constrained to be less than 1.4; consistent with Equation 1.6.2. The mild increase in the X-ray/SFR correlation is predicted from the X-ray binary population synthesis modeling of Fragos et al. (2013) (see Fig. 1). The population synthesis models predict that since higher redshift galaxies have lower metallicities, this enables the production of a more massive and numerous compact object population and more luminous X-ray binaries for a given SFR (see, e.g., Linden et al. 2010). Such a dependence of the $L_{\mathrm{X}} / \mathrm{SFR}$ ratio on metallicity may provide some physical explanation for some of the scatter observed in the local X-ray/SFR correlation. Future studies of both nearby and distant galaxies will be needed to more confidently parameterize the effects of metallicity (and other physical parameters) on X-ray binary emission.

\section{Local Star Formation}

In a Cosmological or Extragalactic context, we are interested in understanding why stars form where they do, whether the efficiency varies, and what factors influence the 
initial mass function (IMF) of the stars, rather than the details of the collapse of a cloud core to a star. Over the last decade, it has become very clear that the star formation rate per co-moving volume was much higher in the past, some 10 or 20 times the current rate at a redshift of $z \sim 0.5-1$ (e.g., Heavens et al. 2004; Wilkins et al. 2008). In turn, this shows that the transformation rate of gas into stars was considerably (factor of a few at least) higher when the universe was roughly half its current age. Galaxies at that time were smaller and of lower metallicity, such that naively at least one would expect that the molecular-to-atomic gas mass ratio would be lower than today (Young et al. 1989; Casoli et al. 1998), making the higher efficiency even more surprising. This may or may not be the case and one of the issues for the next generation of telescopes-ALMA and SKA for the molecular and atomic gas respectively - is the measure of the amount of each type of gas present in "average" spirals out to redshift unity or so. Even for such powerful instruments, it will not be easy to measure the HI, CO, and submillimeter continuum fluxes in order to estimate the gas masses. Furthermore, it is important to spatially resolve the galaxies, separating the central kpc from the disk due to the different gas temperatures and densities, in order to physically interpret the observational results. However, for galaxies in the Local Group, at less than $1 \mathrm{Mpc}$, molecular clouds can be resolved with large antennae and interferometers. Recent studies (e.g., Engargiola et al. 2003; Rosolowsky et al. 2008; Gardan et al. 2007; Gratier et al. 2010; Leroy et al. 2006; Nieten et al. 2006), following the precursor work by Neininger et al. (1998) and Wilson et al. (1997), have provided interesting results, enabling a comparison of the ISM of galaxies of a variety of sizes, morphologies, and metallicities. Fortunately, within $1 \mathrm{Mpc}$ we have a number of small star-forming galaxies-M33, the Magellanic Clouds, NGC 6822, and IC10, in addition to the Milky Way and Andromeda (M31).

An equally interesting alternative is that the IMF has evolved substantially, such that the measured star formation rates simply show that the number of massive stars has increased. The parameter space for this alternative is being reduced due to the number of concordant studies made at different wavelengths and the construction of galaxy Star Formation Histories. Nonetheless, the assumption, often implicit, that the IMF remains constant with cosmological epoch, host galaxy, or local conditions, requires further testing whenever possible.

It is generally believed that the star formation cycle starts with clouds of atomic gas (but see Allen et al. 1997), some of which condenses to form molecular hydrogen $\left(\mathrm{H}_{2}\right)$, from which the stars form in the densest fragments. The most massive stars then create HII regions and photo-dissociation regions (PDRs) and finally disperse much of the cloud. In a way probably linked to the large-scale dynamics (e.g., spiral arms), the scattered material forms clouds anew.

The Atomic gas: The atomic hydrogen is quite extended in galaxies, extending to several times the optical extent in isolated objects. The HI has two stable phases - one warm $(\sim 5000 \mathrm{~K})$ and diffuse and the other cool $(\sim 100 \mathrm{~K})$ and much denser. Presumably it is this phase which is linked to molecular cloud formation and the subsequent stars. Deep high resolution HI measurements, notably by Braun et al. (1997), have shown that the cool phase dominates within the optically bright parts of spirals but that the outer parts (beyond $R_{25}$ ) contain essentially gas in the warm phase. However, the recent GALEX UV observations show that star formation proceeds further out in galactic disks than was believed previously and is spatially correlated with the HI, indicating that there must be a mechanism compressing the warm HI such that it passes into the cool dense phase and then into molecular gas and stars, even where the stellar content is very low. It is not currently clear whether the stellar IMF is necessarily truncated, because the UV is observed further out than $\mathrm{H} \alpha$ emission, or whether high mass stars are rare and 
short-lived enough that their probability of detection is very low (Boissier et al. 2007). Going to smaller, lower metallicity, and more irregular objects, the warm phase appears to dominate throughout as narrow HI line profiles are not seen. In general, molecular gas (see below) is not observed in these objects but star formation is clearly present.

In spirals, the vast majority of the star formation takes place within $R_{25}$ but in isolated objects the rate of flow of gas from the outer disk to the inner disk is not sufficient to replenish the gas gone into stars. Recent very deep observations (Sancisi et al. 2008; Oosterloo et al. 2007) show that extra-planar gas is present in spirals. Does it come from the gradual inflow from past tidal encounters or is it gas that has cooled out from the warm ionized halo gas, becoming neutral and denser than its surroundings and falling towards the disk? The upcoming long-wavelength instruments, SKA and the pathfinders, will extend this work, particularly the southern instruments which have access to the Magellanic Clouds, enabling truly high-resolution observations of the atomic component and how its properties change in the vicinity of molecular clouds.

The Molecular gas: Molecular gas is the direct fuel for star formation. The primary tracer of $\mathrm{H}_{2}$ is the $\mathrm{CO}$ molecule, the most abundant with an electric dipole moment, observed in its rotational transitions at $115 \mathrm{GHz}$ and multiples thereof. A secondary tracer is the continuum emission of the cool dust near the Rayleigh-Jeans part of the spectrum. Because both are metal-dependent, it is unclear to what extent and how these tracers can be used to estimate the $\mathrm{H}_{2}$ mass and thus the Star Formation Efficiency (SFE), the rate of star formation per unit molecular gas, especially in low-metallicity systems. One naturally expects that if the nature of star formation varies with e.g., galaxy morphology, then this will be apparent in the SFE, whether due to a change in the IMF (and thus the estimate of the SF rate) or to a real change in the efficiency of conversion of $\mathrm{H}_{2}$ into stars. It is thus important to properly estimate the $\mathrm{H}_{2}$ mass.

The correlation both in space and velocity between molecular clouds and the $\mathrm{HI}$ is excellent at large scales but breaks down when close to the cloud scale $(\sim 100 \mathrm{pc})$. A major outstanding question is what provokes the transformation of $\mathrm{HI}$ into $\mathrm{H}_{2}$. Comparing the total gas surface density with the SFR as determined from the $\mathrm{H} \alpha$ brightness, Kennicutt et al. (1989) found that a global Schmidt law of $\sigma_{S F} \propto \Sigma_{\text {gas }}^{1.4}$ reproduced the data in his sample and that the Toomre (1964) stability criterion provided a good estimate of the gas surface density threshold for star formation. It now appears clear, with better CO data than was available at the time of those articles, that within individual galaxies the SFR follows the $\mathrm{H}_{2}$ surface density at scales larger than that of GMCs (e.g., Bigiel et al. 2008). Many lines of reasoning point to a threshold $\mathrm{HI}$ column density for $\mathrm{H}_{2}$ and star formation (e.g., Schaye et al. 2004; Martin \& Kennicutt 2001) but so far this has not been unequivocally observed. And it is clear that while $\mathrm{H}_{2}$ (CO in most cases) only forms where HI is present and is more likely to form where HI column densities are higher, for any given (high) HI column density there can be quite a bit of $\mathrm{H}_{2}$ or often none at all. Gardan et al. (2007) showed that in M33 the likelihood of $\mathrm{H}_{2}$ formation per unit gas surface density diminished with radius beyond about $4 \mathrm{kpc}$. In addition to the Toomre criterion, pressure has been proposed (Elmegreen \& Parravano 1994; Blitz \& Rosolowsky 2006) as the factor driving $\mathrm{H}_{2}$ and star formation. While the formalisms proposed provide reasonable results in the bright regions of spirals, they do not predict discrete features such as cut-offs or rings of star formation.

An interesting result, mentioned in passing by Blitz \& Rosolowsky (2006) and Leroy et al. (2006) and focussed on by Gardan et al. (2007) followed by Gratier et al. (2010) and Braine et al. (2010), is that the small, subsolar metallicity, galaxies M33 and IC10 seem to have a higher SFE than the large spirals that dominate the universe today (Murgia et al. 2002; Kennicutt 1998). Dib et al. 2011 have shown that in low metallicity 
environments the stellar winds are weaker, such that molecular clouds stay intact for longer and transform more of their $\mathrm{H}_{2}$ into stars although this is probably not the only cause of the increased SFE. An increase in the SFE is at least partially responsible for the increase in the star formation rate per co-moving volume with redshift (Combes et al. 2012).

While the transformation of $\mathrm{HI}$ into $\mathrm{H}_{2}$ appears to be a medium to large scale process, affected by spiral arms, pressure (related to the stellar surface density), and large-scale compression such as by SN shock waves, the collapse to form stars appears to be a rather local process; this is supported by the apparent constance of the stellar IMF. The basic scenario for low-mass (i.e., solar-like) stars - gravitational collapse of a cool dense core, formation of a disk and bipolar outflows perpendicular to the disk along the magnetic field lines accompanied by an increase of the central temperature, dispersal of disk from stellar winds and arrival of the star on the ZAMS - occurs at a tiny fraction of a parsec. Massive star formation is more problematic as for spherical accretion, the radiation pressure from the protostar should stop stellar growth. Several processes can overcome this problem but require a dense environment and high accretion rates, possibly driven by large scale (1-100 parsec) inflow along the filaments making up large-scale cloud structure. For these reasons, combined with the generally less massive clouds (Gratier et al. 2012; Rosolowsky et al. 2005) and clusters (Corbelli et al. 2011 in the outer parts of spirals, and the idea that the mass of the most massive star in a cluster varies with the cluster mass (Weidner et al. 2011), it appears that massive stars are more easily formed in the inner parts of spirals. Tracing the upper end of the stellar mass function is difficult because these stars have very short main-sequence lifetimes and remain embedded, making it difficult to separate a single very massive star from several less massive stars.

Star formation can be detected in many ways at many different wavelengths - (far) ultraviolet, $\mathrm{H} \alpha$, mid- and far-infrared emission from dust grains, thermal and non-thermal radio continuum emission, as well as other recombination lines and broad-band colors. Some suffer from extinction or require the presence of metals or magnetic fields. It has been known for decades now that star formation generates bipolar outflows. Observing clear signatures of inflow or accretion, although necessary for star formation, has not proved so easy. As such, directly measuring accretion rates or identifying gas inflow is one of the limiting factors in studying star formation and particularly massive star formation.

Galactic and Extragalactic star formation studies are now meeting each other as galactic astronomers try to observe star formation throughout the Galaxy (Schuller et al. 2009; Molinari et al. 2010) and on large scales as extragalactic astronomers resolve star forming regions in nearby galaxies. The latter studies have lower spatial resolution but do not suffer from the distance uncertainties or extinction inherent to studies of our galactic plane. Instruments with high spatial and spectral resolution such as ALMA are expected to contribute greatly to the field of star formation, both by observing protostars in detail and by observing the nearest galaxies with the same linear resolution as earlier studies of the Milky Way.

\section{Initial Mass Functions}

The initial stellar mass function (IMF) determines the number of newly formed stars, $d N$, per (initial stellar) mass interval, $d m$. The IMF is not measurable (Kroupa et al. 2013) but has been inferred on a star-by-star basis only in star clusters and associations, i.e. on parsec scales. Access to the whole stellar mass range down to the regime of brown dwarfs is only possible in the local region of the Milky Way, whereas counting 
massive stars is possible to some extend in nearby Local Group galaxies. Because stars of different masses contribute differently to photometric pass-bands, the knowledge of the functional form as well as the mass limits of the IMF are of fundamental importance for the determination of star formation rates of galaxies. As these galaxies lie beyond the region where star counts are possible, it has to be assumed that the IMF determined in the Milky way can be applied on extragalactic systems.

\subsection{The IMF in correlated star-formation events}

Stars form in the densest regions of molecular clouds. These correlated star-formation events (CSFEs) comprise from a few to millions of stars formed within characteristically 1 Myr and within a region with a radius of less than a pc (Marks \& Kroupa 2012). Some of these CFSEs, commonly also sinply known as embeeded star clusters, emerge to become long-lived, gravitationally bound star clusters.

The empirical IMF inferred from Milky Way CFSEs shows a remarkable universality in its functional form. The form of this universal or canonical IMF is most simply described by a two-part power-law in the stellar regime,

$$
\xi(m)=\frac{d N}{d m} \propto m^{-\alpha_{\mathrm{i}}}
$$

with slopes $\alpha_{1} \approx 1.3$ between $0.07 M_{\odot}$ and $0.5 M_{\odot}$, and $\alpha_{2}=2.3$ above $0.5 M_{\odot}$, where the value of $\alpha_{2}$ is commonly referred to the "Massey-Salpeter" power-law index. Note that this definition of the IMF refers to single objects, i.e. all multiple systems such as binaries or triples are split up to single stars before counting (Kroupa et al. 2013). The upper mass limit is discussed in detail in Sec. 3.3.

Different functional forms are also used in the literature. The most widely used form beside the two-part power law is the log-normal form for stellar masses below $1 M_{\odot}$ but with the same power-law part for $m>1 M_{\odot}$ (Kroupa et al. 2013). This leads to a mathematically more complex object without the gain of physical reality. The physical origin of the form of the IMF is still unclear and subject of theoretical and observational research. But Andre et al. (2010) point out the remarkable similarity between the prestellar core mass function and the stellar IMF, "suggesting a $\sim$ one-to-one correspondence between core mass and star/system mass with $M_{*, \text { sys }}=\epsilon M_{\text {core }}$ and $\epsilon \approx 0.4$ in Aquila."

\subsection{The sub-stellar mass regime}

It has been known for some time that brown dwarfs (BDs) are unlikely to form from direct gravitational collapse in a molecular cloud such that the observationally deduced mass function contains a significant surplus of brown dwarfs (Padoan \& Nordlund 2002, Padoan et al. 2007, Andersen et al. 2011, Hennebelle 2012). Interestingly, several authors have claimed good agreement with the empirical (Chabrier 2003) IMF, but scrutiny of the published work shows consistently significant disagreement (Thies, Pflamm-Altenburg \& Kroupa, in prep.). The reason is that the distribution of density maxima in a cold but turbulent molecular cloud has very few peaks which can collapse through eigengravity at the mass scale of a BD such that not much further material is accreted. Although Withworth et al. (2007) and Whitworth et al. (2010) argue that BDs form a continuous extension of the stellar distribution, the observational and theoretical evidence they provide strongly suggests that BDs and stars have different properties in terms of their pairing (Thies \& Kroupa 2007, Thies \& Kroupa 2008).

Recent work, however, came to the conclusion that the pairing properties of BDs and stars do not show that BDs are significantly different from stars (Reggiani \& Meyer 2011). But this result originates from the usage of the low-mass IMF from Bochanski 
et al. (2010) who fitted a log-normal with $\sigma=0.34$ to a sample of 14 million stars between 0.1 and $0.8 M_{\odot}$. When extrapolating this very tight log-normal function into the BD-regime drawing BDs is highly suppressed and few star-BD binaries are created in Monte-Carlo simulations, in agreement with the observations. On the other hand, when using the IMF which describes the BD-mass regime properly, a significant fraction of BD-star binaries are expected (Kroupa et al. 2003, Thies \& Kroupa 2007, Thies \& Kroupa 2008). The usage of the Bochanski-IMF for BD Monte-Carlo experiments is not understandable as Bochanski et al. (2010) explicitly write in their abstract, "We stress that our results should not be extrapolated to other mass regimes".

Kroupa et al. (2003) have tested the hypothesis that BDs and stars follow the exact same distribution functions and exclude this hypothesis with very high confidence. The various flavours of BDs that can in principle arise (collisional, photo-evaporated, ejected embryos) have been discussed (Kroupa \& Bouvier 2003b) with the result that in the present-day star-forming conditions mostly the ejected embryo flavour dominates. The original suggestion of this scenario has been updated by Stamatellos et al. (2007), Thies et al. (2010), and Basu \& Vorobyov (2012) by the argument that the gravitationally pre-processed material in outer accretion disks is able to cool sufficiently rapidly upon compression to allow direct gravitational collapse at the $\mathrm{BD}$ mass scale. The resulting IMF of BDs compares remarkably well with the observationally deduced BD IMF $\left(\alpha_{0} \approx\right.$ 0.3 ). The resulting binary properties of BDs are also accounted for naturally (Thies et al. 2010).

The BD IMF is thus a nearly flat power-law from the opacity limit for fragmentation $\left(m_{l} \approx 0.01 M_{\odot}\right)$ to an upper limit which transgresses the hydrogen burning limit. In principle, arbitrarily massive "BDs" can form in very massive disks around massive stars such that here the origin of stars vs BDs becomes blurred. Because massive stars are exceedingly rare the stellar population formed through this disk-fragmentation channel is negligible in comparison to the "normal" stellar population which results from direct molecular cloud fragmentation.

Thus in order to correctly account for a stellar population with BDs most of the BD population must be added in terms of a separate distribution function, as is also the case for planets which follow their own mass distribution. The BD IMF can be expressed as a nearly flat power-law with a continuous log-normal extension from the stellar regime being ruled out.

A single continuous log-normal form for the stellar/BD IMF such as the Chabrier form is therefore ruled out by the data.

\subsection{The high-mass end of the IMF - a size-of-sample effect?}

It is widely assumed that the IMF can be interpreted as an environmentally independent probability density distribution function. This IMF has a constant functional form (see Sec. 3.1) and a constant upper stellar mass limit of $\approx 150 M_{\odot}$ (Weidner \& Kroupa 2004, Oey \& Clarke 2005, Figer 2005, Koen 2006). In a recent study Crowther et al. (2010) find that a few massive stars in the very young massive cluster R136 greatly exceed an upper limit of $150 \mathrm{M}_{\odot}$. However, these few outliers can be understood as the stellar merger product in the very dense central region of R136 although the initial stellar masses were smaller than $150 M_{\odot}$ (Banerjee, Kroupa \& Oh 2012).

In this context drawing an $\mathrm{O}$ star from the IMF is much less probable than drawing a low-mass star. As a consequence, in low-mass star clusters the IMF is not fully populated and they are void of massive stars due to the size-of-sample effect. However, serious doubts arose over the last decade that the IMF can be interpreted as a pure invariant probability density distribution function. 
The high-mass slope, $\alpha_{3}\left(m>1 M_{\odot}\right)$, fitted to the set of high-mass stars in individual CSFEs, shows a distribution centred around the Salpeter-Massey index of $\alpha_{3}=2.3$. At first sight this seems to be consistent with the expectation from random drawing. However, despite their youth the observed CSFEs are already dynamically evolved. Therefore, the initial $\alpha_{3}$-spread due to random drawing gets even larger as a result of dynamical evolution. $N$-body simulations of star clusters show that the $\alpha_{3}$-distribution resulting from random drawing and dynamical evolution is broader than the observed distribution of the high-mass slope (Kroupa 2001, Kroupa 2002). There are two possibilities for solving this discrepancy. The predominant finding of a Salpeter-Massey value of $\alpha_{3}=2.3$ is a sociological effect. Or the initial $\alpha_{3}$-distribution must be narrower than expected from random drawing. But this means that the IMF in CSFEs can not be interpreted as a pure invariant probability density distribution function (Kroupa et al. 2013). Instead, if the IMF is a probability distribution function, it must be a constrained one. Or it may not be a probability density function at all.

If the most-massive stars in a CSFE are determined by the size-of-sample effect, as expected from random drawing, then low-mass clusters are typically void of massive stars. Very few CSFEs would exist in which the stellar populations are dominated by one massive O star. Furthermore, some massive stars would then have formed in complete isolation. De Wit et al. (2004, 2005) analyse the Galactic-O-star catalogue and found that 4 per cent of the Galactic $\mathrm{O}$ stars are candidates for massive star formation in isolation. Gvaramadze et al. (2012) analyse these candidates thoroughly. The majority are identified as being runaways based on bow-shock detections and using results from orbit back-tracing from Schilbach \& Röser (2008). The remaining set of candidates is then reduced to one per cent. This little number of O-stars, for which a parent star cluster can not be found, is in agreement with the expectation of two-step ejection, where a massive binary is ejected dynamically from a young star cluster with a subsequent disintegration in the Galactic field due to supernova explosion of the primary. As the new moving direction of the secondary is arbitrary runaway can not be traced back to its parent star cluster (Pflamm-Altenburg \& Kroupa 2010). There is therefore no significant evidence that massive stars can form in isolation.

If the most-massive-star in a CSFE is determined by the size-of-sample effect than a relation between the mass of the most-massive stars and the total stellar mass of the CSFE with a broad scatter emerges. In order to compare this prediction with the observations an unbiased set of CSFEs is required and are selected by the following two criteria: i) All star clusters must be younger than $\approx 4$ Myr in order to exclude star clusters where the most-massive star has already exploded in a supernova (such clusters would have a less massive most-massive star). ii) All star clusters must be still embedded in their natal gas (otherwise the star clusters can have already expanded due to the effect of gas expulsion (Kroupa et al. 2001) and may have lost a significant fraction of their stars and are therefore reduced in mass). A thorough analysis by Weidner \& Kroupa (2006) and Weidner et al. (2010) shows that the observed relation between the most-massive star and the stellar mass of the CSFE lies statistically significantly below the predicted relation from the random sampling ansatz, therewith ruling out an invariant probability density distribution interpretation of the IMF. Considering dynamical ejections of the most-massive stars, this conclusion is not affected (Oh \& Kroupa 2012, Pflamm-Altenburg - in prep.).

Contrary to the results above, Maschberger \& Clarke (2008) came to the conclusion that "the data are not indicating any striking deviation from the expectations of random drawing". But they restricted their analysis to the low-mass star cluster data from Weidner \& Kroupa (2006) and complemented them with additional low-mass star clusters 
including the data set from Testi et al. (1997, 1998). After finding a KS-probability of $10^{-14}$ for an agreement with random sampling they removed the Testi-data from their sample. After subsequently reducing the sample even further they finally end up obtaining a KS-probability of 20 per cent for an agreement with random sampling. But, the need of giving up the picture of a randomly sampled constant IMF described above is driven by the result from the whole data of most-massive stars in star clusters and not only by a tiny subset of low-mass star clusters as employed by Maschberger \& Clarke (2008). Furthermore, they have tested no alternatives. And finding that model A explains the observations does not imply that model B is immediately shown to be wrong. It should be mentioned here that the work by Maschberger \& Clarke (2008) has been wrongly cited by Lamb et al. (2010). They write: "Maschberger $\&$ Clarke (2008) complement the WK06 data set with a sample of very small clusters from Testi et al. (1997) and find that the resultant ensemble of clusters does not significantly deviate from the expectations of a universal stellar IMF, when examining the correlation between the number of stars in a cluster $(N)$ and $m_{\max }$ ". Such false citations to work which selects data in order to achieve a pre-conceived result, as seems to be the case, lead to appreciable damage to scientific progress.

The observational result that the formation of massive stars require a compact highmass environment is supported by the recent work by Hsu et al. (2012). They examine the young stellar content of the low-density star-forming region L 1641 in the Orion A cloud and find that it is deficient in $\mathrm{O}$ and $\mathrm{B}$ stars to a 3-4 $\sigma$ significance level. This is in-line with the result from hydrodynamical star-formation simulations, where the upper mass limit of stellar groups scales with the number of stars of these subgroups (Maschberger et al. 2010).

We can finally summarise that there is very strong evidence that the IMF can not be treated as a pure invariant probability density distribution function and that the formation of high-mass stars is not characterised by the size-of-sample effect but requires a massive and compact. ie. dense, environment.

\subsection{IMF variations under extreme conditions}

The above discussion considers the IMF in young star forming regions in the Milky Way. The functional slope of the IMF seems to be universal whereas the population of the high-mass regime can not be driven by pure statistics. However, recent analyses of observations show that the functional form of the IMF may have been different under extreme conditions, which are not present in the star forming regions in the vicinity of the Milky Way considered above.

Two indications exist that the IMF may have been bottom-heavy in cases of high metallicity: i) A hint at a possible variation of the IMF in the MW has emerged due to present-day star-formation events possibly producing more low-mass stars than previously. This has been quantified as a metallicity dependence, $\alpha \approx 1.3+0.5[\mathrm{Fe} / \mathrm{H}]$ (Kroupa et al. 2013). ii) From the study of massive elliptical (E) galaxies, it has emerged that the IMF may have been significantly bottom heavy when the E galaxies formed. Based on spectral analyses Cenarro et al. (2003) inferred $\alpha=3.41+2.78[\mathrm{Fe} / \mathrm{H}]-3.79[\mathrm{Fe} / \mathrm{H}]^{2}$ (for $0.1<m / M_{\odot}<100$, although not explicitly stated in the paper). iii) A more recent analysis by van Dokkum \& Conroy $(2010,2011)$ also suggests an increasingly bottom heavy IMF with increasingly massive E galaxies. This may be related to the postulated cooling-flow-accretion population of low-mass stars (Kroupa \& Gilmore 1994).

An unusual IMF in massive ellipticals is also reported by Cappellari et al. (2012) based on their larger mass-to-light ratios. The authors conclude that this can be due 
to an bottom-heavy IMF (enriched by faint low-mass stars) as well as a top-heavy IMF (enriched by non-luminous remnants of high-mass stars).

Whitworth et al. (1994) had already suggested that massive stars may preferentially form in shocked gas. As reviewed in Kroupa et al. (2013) there has been much observational evidence for top-heavy IMFs in star-bursts. As these are observationally unresolved, this evidence was indirect and largely ignored. Observations of the assembly of the stellar population over cosmological epoch have also been pointing to top-heavy IMFs in the cosmological past, as otherwise there would be more low-mass stars locally than are observed. Three independent more-direct lines of evidence for the IMF becoming top-heavy with star-formation rate density have recently emerged:

Firstly: It is well known that ultra-compact dwarf galaxies (UCDs), which have a mass scale of $10^{6}-10^{8} M_{\odot}$, have larger dynamical mass-to-light (M/L) ratios than normal stellar populations. This is unlikely due to exotic dark matter as the phase-space available in UCDs would not accommodate significant amounts of dark matter. Instead, a topheavy IMF would have lead to an overabundance of stellar remnants in UCDs which would enhance their dynamical $\mathrm{M} / \mathrm{L}$ ratios. Thus, the variation of the required $\alpha_{3}, m>$ $1 M_{\odot}$, can be sought to explain the dynamical M/L ratia (Dabringhausen et al. 2009).

Secondly: A larger-than-expected fraction of UCDs have low-mass X-ray bright sources (LMXBs). In globular clusters (GCs), LMXBs are known to be formed from the dynamical capture of stars by stellar remnants mostly in the core of the GCs. As the star evolves the remnant accretes part of the star's envelope thus becoming detectable with X-rays. The LMXB population is constantly depopulating and needs to be replenished by new capture events. Indeed, the theoretically expected scaling of the fraction of GCs with LMXB sources with GC mass is nicely consistent with the observed data assuming an invariant stellar MF. Applying the same theory to UCDs uncovers a break-down of this agreement as the UCDs have a surplus of LMXB sources. By adding stellar remnants through a top-heavy IMF when the UCDs were born, i.e. by allowing $\alpha_{3}$ to vary with UCD birth mass, consistency with the data can be sought (Dabringhausen et al. 2012).

Thirdly: Low-concentration GCs have been found by de Marchi et al. (2007) to be depleted in low mass stars while high-concentration GCs have a normal MF. This is contrary to the energy-equipartition driven depopulation of low mass stars because more concentrated clusters ought to have lost more low mass stars. It is also not consistent with any known theory of star formation, because the low-concentration clusters are found to typically have a higher metallicity which would, if anything, imply a surplus of low-mass stars. The currently only physically plausible explanation is to suppose that the young GCs formed compact and mass segregated and that the expulsion of residual gas unbound a part of the low-mass stellar population. By constraining the necessary expansion of the proto-GCs, correlations between metallicity, $\alpha_{3}$ and tidal field strength emerge which constrain the very early sequence of events that formed the Milky Way as well as the dependency of $\alpha_{3}$ on density and metallicity of the CSFE which can lead to a bound long-lived globular cluster after emergence from the natal cloud (Marks et al. 2012).

Putting all this together (Marks et al. 2012, Kroupa et al. 2013), a consistent variation of $\alpha_{3}$ with density and metallicity of the CSFEs emerges: for $m>1 M_{\odot}, x \geqslant-0.89$ : $\alpha_{3}=-0.41 x+1.94$ with $x=-0.14[\mathrm{Fe} / \mathrm{H}]+0.99 \log _{10}\left(\rho_{6}\right)$, where $\rho_{6}=\rho /\left(10^{6} M_{\odot} \mathrm{pc}^{-3}\right)$ and $\rho$ is the density in $M_{\odot} / \mathrm{pc}^{3}$. This translates into a star-formation rate density (SFRD) assuming the CSFEs form within $1 \mathrm{Myr}$.

Thus, CSFEs with $S F R D<0.1 M_{\odot} /\left(\mathrm{pc}^{3} \mathrm{Myr}\right)$ can be assumed to have an invariant IMF with $\alpha_{3}=\alpha_{2}$ (subject to the possible variation with metallicity discussed above), 
while CSFEs with larger SFRDs tend towards top-heavy IMFs whereby the trend is enhanced at lower-metallicities.

\subsection{The IGIMF}

The previous sections consider the stellar content in correlated star-forming events (CSFEs), i.e. essentially in embedded star clusters which need not be gravitationally bound on emergence from their natal clouds. But when converting galaxy-wide photometric and spectroscopic data into physical properties (e.g. SFRs) the galaxy-wide IMF has to be known.

As stars form in CSFEs, i.e. in embedded star clusters or clusterings (Lada \& Lada 2003, Allen et al. 2007), the galaxy wide-IMF has to be calculated by adding all IMFs of all CSFEs in a galaxy, irrespectively of whether the distribution functions are pure probability density functions or whether they are, at the other extreme, optimally sampled distributions (Kroupa et al. 2013). This is the basic tenet of the integrated galactic initial mass function (IGIMF) theory (Kroupa \& Weidner 2003, Weidner et al. 2004).

In deriving the analytical IGIMF theory, the following empirical star formation laws need to be considered: (i) The functional form of the IMF in each CSFE is invariant for $S F R D<0.1 M_{\odot} /\left(\mathrm{pc}^{3} \mathrm{Myr}\right)$, becoming top-heavy for lager values of $S F R D$ (Sec. 3.4). (ii) The most-massive star in each CSFE scales with the stellar mass of the CSFE according to the observations (Sec. 3.3). (iii) Observations show that the mass of the mostmassive CSFE (i.e. young star cluster) scales with the current SFR of a galaxy (Weidner et al. 2004). This means that low-mass CSFEs are void of high-mass stars, and low-SFR galaxies do not host high-mass CSFEs.

The combination of these empirical relations leads to an IGIMF which differs from the canonical IMF in a CSFE. The IGIMF becomes steeper in the stellar high-mass regime with decreasing SFR (Weidner \& Kroupa 2005, Pflamm-Altenburg et al. 2007). Thus with decreasing SFR the fraction of high-mass stars among all newly formed stars decreases, which is called the IGIMF-effect. At high galaxy-wide SFRs, the IGIMF becomes topheavy relative to the canonical IMF. At low SFRs the IGIMF becomes top-light relative to the canonical IMF.

Consequently, as low-SFR galaxies have a smaller fraction of ionising stars, these galaxies have a higher SFR-H $\alpha$-luminosity ratio than Milky Way type galaxies (PflammAltenburg et al. 2007). Thus, if using a classical linear SFR-H $\alpha$-luminosity relation the SFRs of dwarf galaxies are underestimated by up to two orders of magnitude. Correcting the obtained SFRs for the IGIMF effect it emerges that dwarf galaxies are not inefficient in forming stars but have the same star-formation efficiency as Milky-Way type galaxies. That is, it emerges that the SFR scales linearly with the gas mass of the galaxy (Pflamm-Altenburg et al. 2009) and that galaxies have constant gas depletion time-scales of $\approx 3$ Gyr.

In contrast to the $\mathrm{H} \alpha$ luminosity which has its origin in the presence of ionising highmass stars, long-lived B-stars contribute most to the far ultraviolet (FUV) luminosity. Thus the IGIMF-effect is very much stronger for $\mathrm{H} \alpha$-radiation than for the FUV-flux. This has been predicted in Pflamm-Altenburg et al. (2009) and is in remarkable agreement with the observations by Lee et al. (2009).

The IGIMF-model can be refined into a local surface-density framework, where the mass of the locally most-massive CSFE scales with the local gas density. This formulation of the local IGIMF (LIGIMF) explains simultaneously the observed $\mathrm{H} \alpha$ cut-off in the disks of star forming galaxies and their extended FUV-disks (Pflamm-Altenburg \& Kroupa 2008). 
Yields of chemical elements are effected by the IGIMF-effect as well. As the fraction of high-mass stars decreases with decreasing SFR the effective oxygen yield should decrease (Köppen et al. 2007). This automatically leads to a mass-metallicity relation of galaxies as observed (Tremonti et al. 2004). In order to explain the observed mass-metallicity relation of galaxies in terms of an invariant IMF, metal enriched outflows must be postulated to be active in dwarf galaxies to account for the required low effective oxygen yields. On first sight this might be plausible as lower-mass galaxies have shallower gravitational potentials than large disk galaxies, and expanding supernova shells containing freshly produced metals might escape easier from dwarf galaxies. In order to break the degeneracy between the IGIMF-model, on the one-hand side, and the constant IMF model plus metal enriched outflows, on the other hand side, one has to concentrate on galaxies with different SFRs but equal gravitational potentials. This can be done by comparing lowsurface brightness galaxies (LSBs) with normal disk galaxies having the same rotational velocity which is a proxy for the deepness of the gravitational potential. For the same potential, the constant-IMF model combined with metal enriched outflows would predict that the effective yields are higher for LSBs than for normal disk galaxies, because normal disk galaxies have higher SFRs and larger feedback by supernovae and metals should escape easier reducing the effective, i.e. detectable, yields. The IGIMF-model, on the other hand, predicts that the effective yields are higher for normal disk galaxies than for LSBs because normal disk galaxies have higher SFRs and therefore flatter IGIMFs and a larger fraction of high-mass stars. An analysis of effective oxygen yields of highand low-surface brightness galaxies show that the IGIMF model is in agreement with the observations (Pflamm-Altenburg et al. 2011).

Furthermore, as Fe and $\alpha$-elements have their main contribution from supernovae for which the stellar progenitors have different masses, the IGIMF-effect predicts different yields for both types of elements. Observations show that the $[\alpha / \mathrm{Fe}]$ abundance ratio decreases with decreasing velocity dispersion of early type galaxies. As lower-mass elliptical (E) galaxies had smaller SFRs than high-mass E galaxies, the production rate of $\alpha$-elements should decrease faster with decreasing velocity dispersion than the production rate of Fe. Recci et al. (2009) show that the observations are in agreement with the expectation from the IGIMF-theory.

\subsection{The IGIMF - ruled out?}

Despite the most remarkable success of the IGIMF theory to naturally explain a large variety of observational results, a number of authors claim to have falsified the theory. These works are often cited by others uncritically as evidence against the IGIMF theory. Here we briefly touch upon these works noting that a more thorough analysis will be presented elsewhere.

The successful prediction of the $\mathrm{H} \alpha / \mathrm{UV}$ luminosity ratio in dwarf galaxies by the IGIMF theory has been challenged recently. Fumagalli et al. (2011) made Monte-Carlo simulations of star forming galaxies by randomly sampling both distribution functions: the star cluster mass function (CMF) of a star-forming galaxy and the IMF in each star cluster. Therewith they unknowingly apply the basic tenet of the IGIMF theory because they assume the IGIMF to be the result of the integration over all star clusters. They nevertheless conclude "that a truncation in the IMF in clusters is inconsistent with the observations". But the description of their publicly available code (da Silva et al. 2012) reveals that the relation of the most-massive star and the mass of the star cluster has been implemented as a truncation limit of the IMF and not as the observed most-massive star of the particular star cluster. This means that the additional under-sampling effect 
in their calculations leads to wrong results. The IGIMF has been implemented wrongly by Fumagalli et al. (2011).

Calzetti et al. (2010) tested if low-mass star clusters are void of very massive-stars due to physical constraints, as stated by the IGIMF theory which in turn is based on the best observational material available from fully resolved star-forming regions, rather than being purely statistical, as would be the case if the IMF and IGIMF were identical and pure probability density distribution functions. In order to perform their test, they used extragalactic observations: if the upper mass regime of the IMF in star clusters is populated according to the size-of-sample effect then low-mass clusters must have the same $\mathrm{H} \alpha$-luminosity/star-cluster-mass ratio as high-mass star clusters if the sample of low-mass star clusters is sufficiently large. They came to the conclusion that their "results for NGC5194 show no obvious dependence of the upper mass end of the IMF on the mass of the star cluster down to $\approx 10^{3} M_{\odot}$ ". However, a thorough perusal of their Figure 1 shows that the $1 \sigma$ error ellipse is slightly closer to the IGIMF-model than to the expectation of pure random sampling. Other issues with this work by Calzetti et al. (2010) will be published soon, and it is clear that their statements are based on an incorrect account of the biases at work in extragalactic observations of individual star clusters.

Weisz et al. (2012) compare the spread of $\mathrm{H} \alpha$ luminosities of galaxies with the same FUV-luminosity. They argue that "these results demonstrate that a variable IMF alone has difficulty explaining the observed scatter in the Ho-to-FUV ratio". They favour that the variability in the star formation history is responsible for the observed spread. However, Weisz et al. (2012) utilize a fully populated IMF, which is scaled to match the observed luminosities. This means that even in low-star formation rate galaxies, where the classical picture of the IMF requires the size-of-sample effect, their IMF is fully populated. In their model, dwarf galaxies would have fractions of $\mathrm{O}$ stars with the corresponding fraction of their hard-photon flux, which is unphysical. Thus again, this approach at testing the IGIMF theory suffers from an unphysical approach.

Taking the analytical IGIMF theory, according to which all distribution functions are optimally sampled density distributions following the empirical correlations stated above, then the predictions of this theory are precise. In comparing with observational data, such as extragalactic ones, it needs to be remembered that the observational data always carry observational uncertainties (random errors) and biases (systematic errors) which can never be removed. Thus, an observational ensemble of star-forming galaxies will always show a significant scatter which may, however, be unphysical.

The IGIMF-model as an analytic formulation can not account for fluctuations in the upper mass regime of the IMFs in star clusters (e.g. through stochastically occurring stellar mergers). An extended formulation of the IGIMF-theory may be capable to predict physical spreads of $\mathrm{H} \alpha$ luminosities of galaxies in the future. But first it has to be clarified how the upper mass regime of the IMF is populated and which physical processes are responsible for the spread in the high-mass regime of the IMF. The only clear fact at the moment is that this regime can not be populated according to the size-of-sample effect, that is, the IMF is not a pure probabilistic distribution function (Sect. 3.3). As such, the analytical IGIMF theory is based on the observed relation of the most-massive star in a CSFE as a function of the CSFE stellar mass, and on the observed relation of the most-massive young CSFE as a function of the current SFR of a galaxy. These relations determine the typical mass of the most-massive star in a CSFE and the typical mass of the most-massive young star cluster in a galaxy. Consequently, the analytical IGIMF-theory specifies the typical galaxy-wide IMF of a galaxy for a given SFR. 
Nevertheless, the published papers discussed here (e.g. Fumagalli et al. 2011, Sharma et al. 2011, Relaño et al. 2012) are wrongly misused as evidence against the basic concept of the IGIMF theory. Detailed perusal of this published work shows that so far there is no rigorous evidence against the IGIMF-theory as being the correct description of star formation in galaxies.

\section{SFR determinations from SED modelling}

During this meeting we have discussed a lot about different methods of deriving star formation rates in galaxies and what the advantages and disadvantages of using different calibrations are (see the comprehensive review talk by Veronique Buat at this meeting; see also review by Calzetti 2012). During the session on SFR determinations from SED modelling, but also throughout the meeting, two SED modelling approaches have been presented and discussed: the energy balance method and the radiative transfer modelling.

The energy balance method relies on the conservation of energy between the stellar light aborbed by dust and that emitted (by the same dust) in the mid-IR/far-IR/submm. This is by far a superior method to only fitting SED templates in a limited spectral range (see talk by Denis Burgarella). Nonetheless, the energy-balance method is not a self-consistent analysis, since the SED of dust emission is not calculated according to the radiation fields originating from the stellar populations in the galaxy under study, but rather according to some templates. The templates can be either empirical (Xu et al. 1998, Devriendt et al. 1999, Sajina et al. 2006, Marshall et al. 2007) or theoretical (Dale \& Helou 2002, Draine \& Li 2007, Natale et al. 2010). At this meeting we saw applications of two energy balance methods, MAGPHYS (da Cuhna et al. 2008) and CIGALE (Burgarella et al. 2005, Noll et al. 2009). The energy balance method is a useful tool when dealing with large statistical samples of galaxies for which little information is available regarding morphology/type, orientation and overall size. This advantage comes nevertheless with the disadvantage that the energy balance methods cannot take into account the effect on the dust attenuation and therefore also on the dust emission of the different geometries of stars and dust present in galaxies of different morphological types, neither can it take into account the anisotropies in the predicted stellar light due to disk inclination (when a disk geometry is present). These methods can therefore only be used in a statistical sense, when dealing with overall trends in galaxy populations.

The radiative transfer method is the only one that can self-consistently calculate the dust emission SEDs based on an explicit calculation of the radiation fields heating the dust, consequently derived from the attenuated stellar populations in the galaxy under study. At this meeting we saw applications of the RT model of Popescu et al. (2011). This method can take advantage of the constraints provided by available optical information like morphology, disk-to-bulge ratio, disk inclination (when a disk morphology is present) and size. As opposed to the energy balance method, this advantage comes with the disatvantage that such information is not always easily available. Nonetheless these radiation transfer methofs could perhaps be adapted to incorporate this information (when missing) in the form of free parameters of the model, though such attempts have not yet been made. Another drawback of these methods was that radiative transfer calculations are notorious for being computationally very time consuming, and as such, detailed calculations have been mainly used for a small number of galaxies (Popescu et al. 2000, Misiriotis et al. 2001, Popescu et al. 2004, Bianchi 2008, Baes et al. 2010, MacLachlan et al. 2011, Schechtman-Rook et al. 2012, de Looze et al. 2012a,b). This situation has been recently changed, with the creation of large libraries of radiative transfer model SEDs, as performed by Siebenmorgen \& Krugel (2007) for starburst galaxies, 


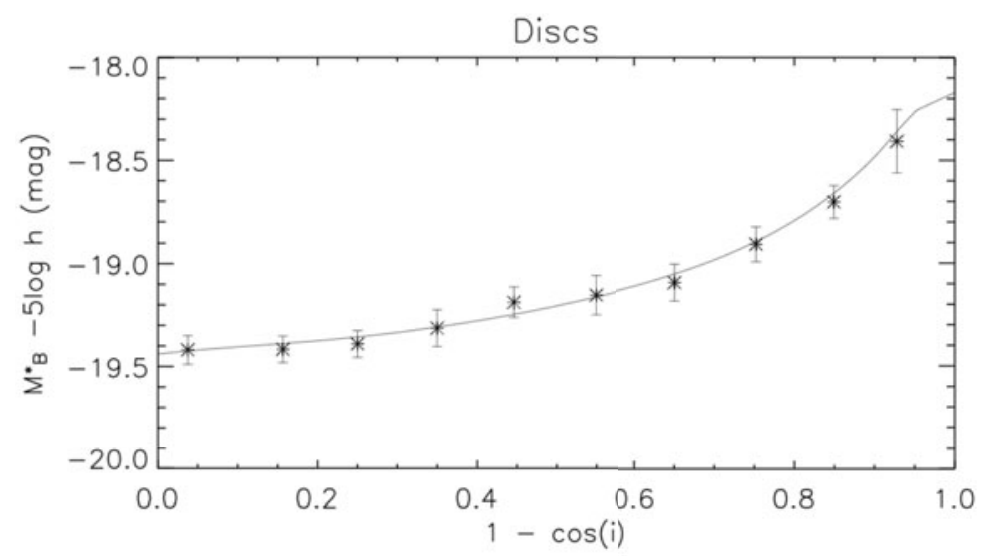

Figure 9. The attenuation-inclination relation from Driver et al. (2007). The symbols deliniate the empirical relation derived for disks from the Millenium Galaxy Survey while the solid line is the prediction from the model of Tuffs et al. (2004).

Groves et al. (2008) for star-forming regions/starburt galaxies and Popescu et al. (2011) for spiral galaxies.

Reviews on determination of star-formation in galaxies have so far not included discussions on the use of radiative transfer methods, with the exception of the review of Kylafis \& Misiriotis (2006). With the new developments resulting in the creation of libraries of RT models, we can now start to include radiative models as main topics of discussion. Indeed, at this meeting we emphasised that they are in fact the most realible way of deriving star formation rates in galaxies. In this way the SFRs are derived self-consistently using information from the whole range of the electromagnetic spectrum, from the UV to the FIR/submm, incorporating information with morphological constraints (primarily from optical imaging). Here we did not consider radio and Xray emission, though these emissions have been discussed in other sessions of this meeting (e.g. talk by Bret Lehmer). In particular the SED modelling has been discussed in conjunction with the most difficult cases, namely those of translucent galaxies: galaxies with both optically thin and thick components. In one way optically thin galaxies are more easily dealt with, since most of the information on SFR can be derived from the UV. Very optically thick cases are also easy from this point of view, since SFR can be derived from their FIR emission, providing one can isolate the AGN powered emission. But the most difficult cases are the translucent galaxies. These are essentially the spiral galaxies in the Local Universe, and probably a large fraction of the star forming dwarf galaxies - which dominate the population of galaxies in the Local Universe, and which also host most of the star formation activity taking place in the local Universe. We showed in this meeting how important it is to quantify this star formation activity. We also discussed how important it is to quantify the star formation activity in the high redshift Universe; it is just that for the moment we do not have enough detailed information to be able to do the same type of analysis that we can now do for the Local Universe. Here I will summarise the main points we addressed:

1. Why is it so difficult to calibrate SFR in spiral galaxies and why do we need to follow the fate of photons with radiative transfer calculations?

- Fundamentally, any fixed observed luminosity in dust emission can be powered either by a small fraction of a large quantity of optical light from older stellar populations, or 
by a large fraction of a small quantity of UV light from younger stellar populations. Only radiation transfer techniques can unravel this dichotemy.

- Because of the disky nature of these systems, the direct UV and optical light is highly anisotropic, and the attenuation of the stellar photons will depend on the viewing angle and wavelength. Fig. 9 illustrates the strong dependence of the observed luminosity of stellar disks on the viewing angle.

- Dust in galaxies has a very complex structure, containing both diffuse components on kiloparsec scales, as well as localised components, at the pc scales, associated with the star forming regions. The escape of radiation from these two components is very different, as it is the heating of dust in the diffuse medium and in the star-forming clouds.

- Disk galaxies have different morphological components, in particular disks and bulges. The attenuation characteristics of disks is very different from those of bulges (see Fig. 10) and these need to be properly taken into account when dealing with the integrated emission from galaxies.

- Different stellar populations have different spatial distributions with respect to the dust distribution, and again their attenuation characteristics will differ, as will their contribution to heating the dust. Fig. 10 illustrates the different behaviour of the variation of attenuation of light coming from different stellar populations with inclination and dust opacity. Fig. 11 also shows how the dust and PAH emission SEDs are changed for various contributions coming from the old and young stellar populations in the disk, as well as from the old stellar populations in the bulge.

\section{Why do SFR calibrators work?}

SED modelling tools based on self-consistent radiative transfer calculations can be used to predict the scatter in the SFR calibration relations, as a function of the main intrinsic parameters that can affect these relations. Several of these relations have been presented. In Fig. 12 we only show predictions for the SFR calibration based on monochromatic FIR luminosities when the dust opacity changes. The predictions are based on the model of Popescu et al. (2011). The figure shows a very large scatter in the correlations. A similar large scatter is predicted for correlations corresponding to various contributions coming from the old stellar populations, or for the clumpiness of the ISM. For the UV calibrators large scatters are also predicted when some of the relevant parameters (viewing angle, dust opacity, bulge-to-disk ratio and clumpiness of the ISM) vary. Overall it is apparent from these plots that the predicted scatter in the SFR correlations due to a broad range in parameter values is larger than observed in reality. The question then arises of why are the SFR calibrators working, despite, for example, the very crude dust corrections that have been so far used in the community? A possible answer is the existence of some scaling parameters, which do not allow a continuous variation in parameter space, in particular for dust opacity or stellar luminosity. Recent work from Grootes et al. (2013) proved the existence of a well-defined correlation between dust opacity and stellar mass density. The correlation was derived on data coming from Galaxy and Mass Assembly (GAMA) survey (Driver et al. 2011) and the Herschel ATLAS survey (Eales et al. 2011), in combination with the model of Popescu et al. (2011). These finding give support to the interpretation of the existence of fundamental physical relations that reduce the scatter in the SFR correlations.

\section{A word of caution}

We have identified some points where things should be treated more carefully in the future:

- The energy balance method should not be used on scales smaller than the scalelength 

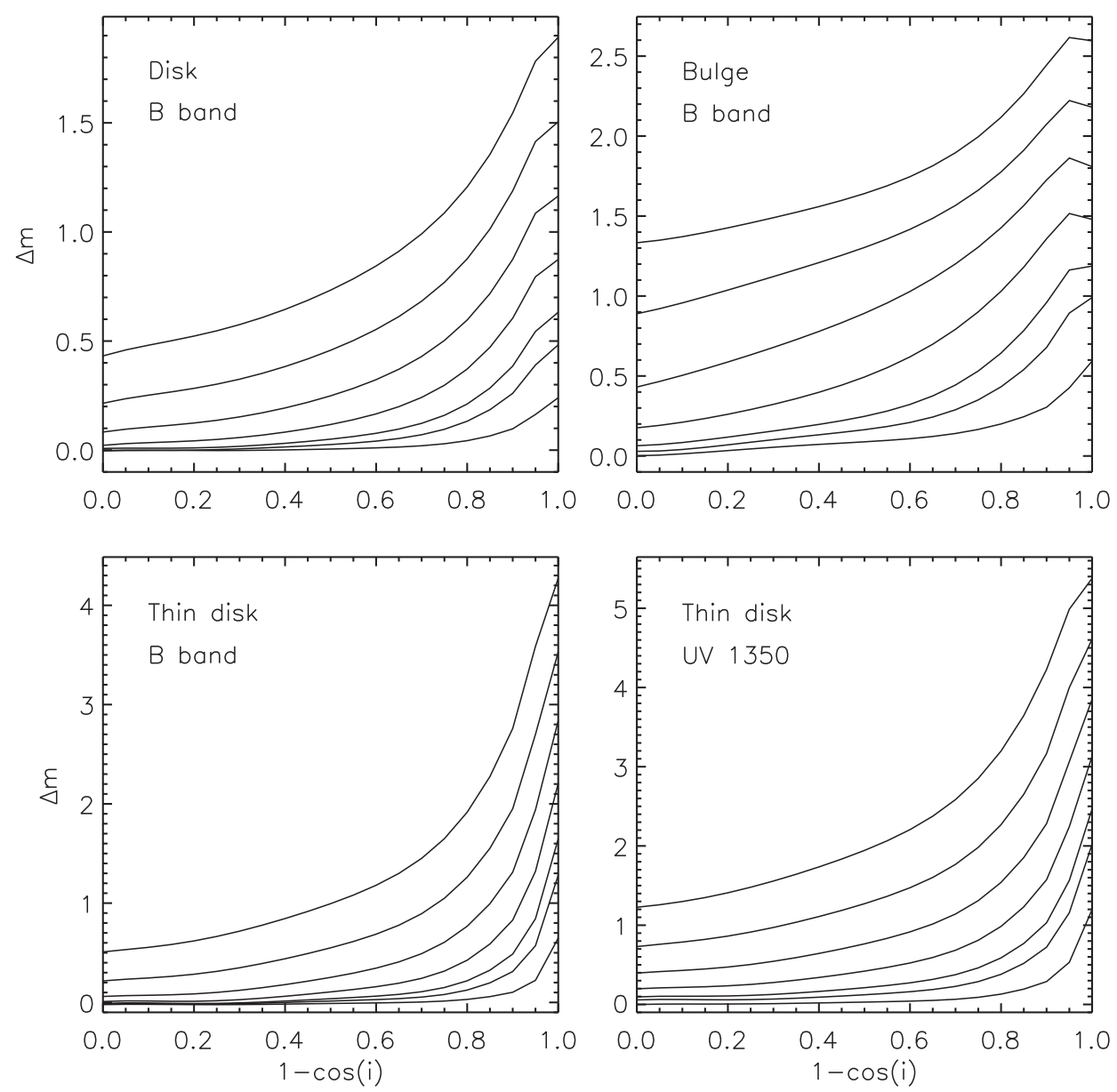

Figure 10. Predictions for the attenuation-inclination relation for different stellar components from Tuffs et al. (2004). From bottom to top the curves correspond to central face-on B band optical depth $\tau_{B}^{f}$ of $0.1,0.3,0.5,1.0,2.0,4.0,8.0$

of the disk, as the energy is not conserved below these scales. The role of long range photons in the diffuse ISM should not be underestimated, in particular by considering an average free path of photons in the disk. This is because the free path of photons firstly depends on radial position in the galaxy (disk), where dust opacity is known to decrease monotonically with radius (e.g. Boissier et al. 2004, Popescu et al. 2005). Secondly, there is also a vertical distribution of dust, and the free path of photons in vertical direction will be different from that in radial direction. One also needs to add the contrast between arm and interarm regions. Finally, the escape of photons from star-forming clouds is strongly anisotropic and fragmented, because of the fragmentation of the clouds themself. Thus, in some directions the stellar light is completely absorbed by dust, while there are lines of sight from which the radiation freely escapes in the surrounding diffuse medium. The multiple facets of the transfer of radiation in galaxies, including the effect of scattered light, means that energy balance method should not be applied on a pixel by pixel basis, as sometimes employed in the literature.

- Mid-IR emission should not only be identified with "small grain" emission, where by small grain we mean stochastically-heated grains. In fact in the range $24-60 \mu \mathrm{m}$ most 

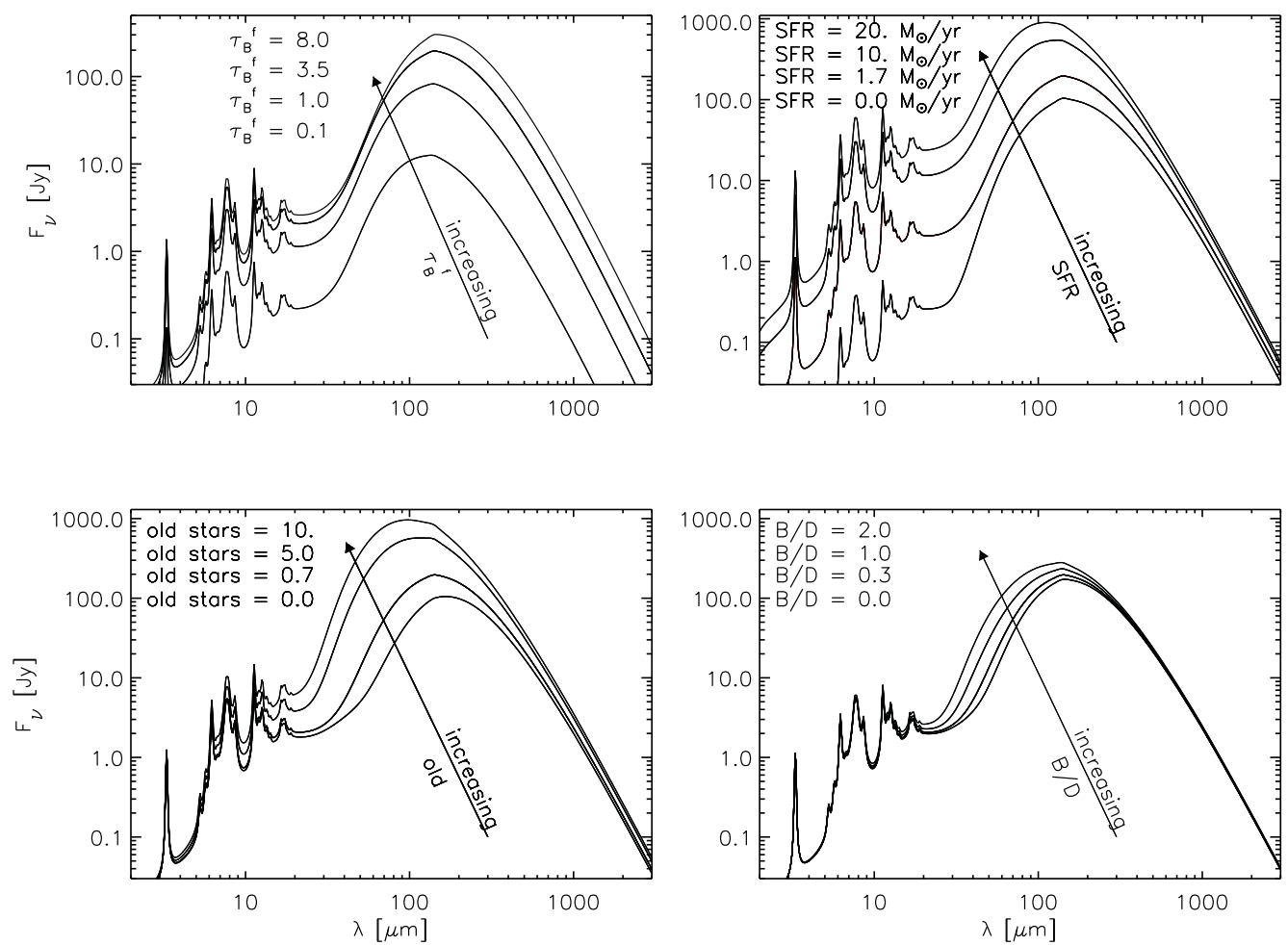

Figure 11. Predictions for dust and PAH emission SEDs based on the model of Popescu et al. (2011). In going clock-wise from the top-left, the different panels show the effect of changing the dust opacity, the luminosity of the young stellar populations (SFR), the luminosity of the old stellar populations (old) and the bulge-to-disk (B/D) ratio. In each panel only one parameter at a time is changed, while keeping the remaining ones fixed.
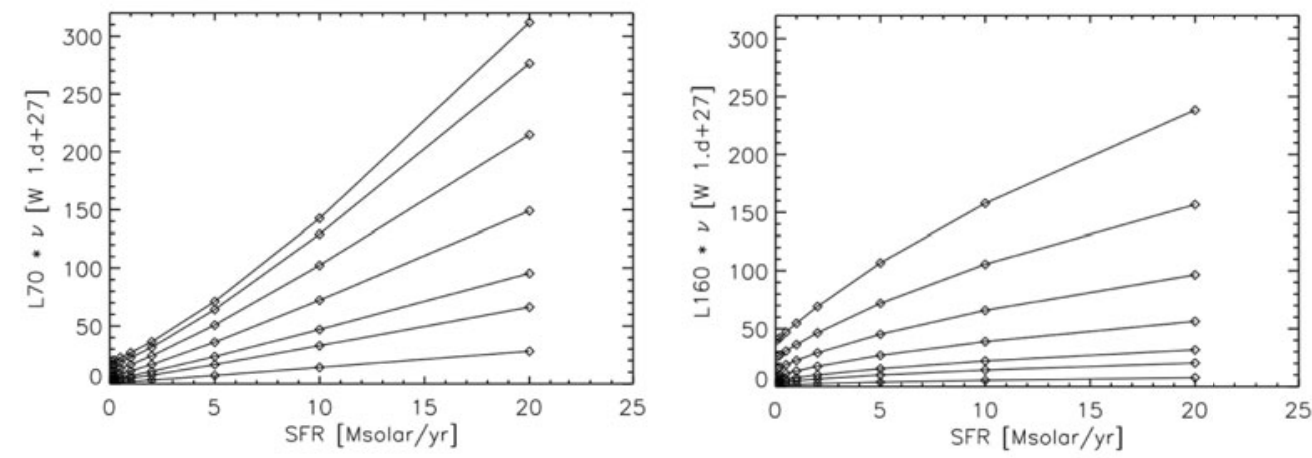

Figure 12. Predictions for the relation betwween the $70 \mu \mathrm{m}$ (left) and $160 \mu \mathrm{m}$ luminosity versus SFR based on the model of Popescu et al. (2011). From bottom to top the curves correspond to central face-on B band optical depth values of 0.1,0.3,0.5,1.0,2.0,4.0 and 8.0.

of the dust emission is powered by big grains heated at equilibrium temperatures by the strong radiation fields in the star-forming complexes.

- The ratio between mid-IR (PAH range) emission to FIR emission cannot be interpreted only in terms of relative abundances of PAH to big grains. One should also take 

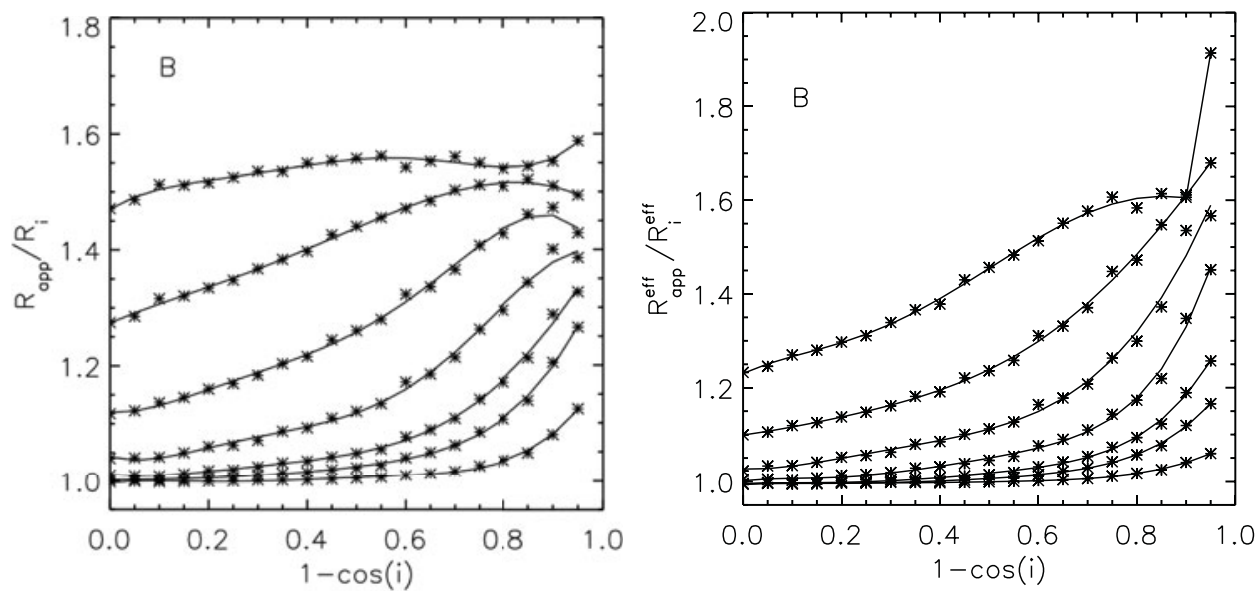

Figure 13. Dust effects on the derived scalelength of disks fitted with exponential functions (left) and on the derived effective radius of disks fitted with variable index Sérsic functions, from Pastrav et al. (2013). Both plots are for the B band. From bottom to top curves are for a central face-on dust opacity in the B band of $0.1,0.3,0.5,1.0,2.0,4.0,8.0$.

into account the change in the colour and intensity of the radiation fields heating the dust, which also result in strong variations of mid-IR to FIR emission.

- Do we really need to accurately know the absolute star-formation rates in galaxies? Perhaps we can live with some approximations, which would be good enough to allow us to derive trends in galaxy populations over cosmic time. A definitive no has been given to this suggestion. An inability to measure absolute SFR would severely limit our ability to constrain physical models of galaxies and the evolving universe. For example, only if we have absolute measurements of SFR will we be able to relate measurements of SFR to measurements of gas content of galaxies in terms of physical models predicting the amount of gas in the ISM and the efficiency of conversion of the ISM into stars. Pavel Kroupa also gave convincing statistical argumentation on the need to measure accurate SFRs in the discussion session.

\section{What else have we learned?}

- Applications of self-consistently calculated model SEDs strongly rely on scaling them according to measurements of the surface area of the stellar disk of the modelled galaxy, which, in turn, depends on an accurate decomposition of the main morphological components of galaxies as observed in the UV/optical. Bogdan Pastrav showed that the derived scale-sizes of stellar disks of galaxies are strongly affected by dust (see Fig. 13), and that a proper determination of the intrinsic distributions of stellar emissivity, and thus of star-formation rates, needs to self-consistently take into account these effects.

- Several panchromatic surveys, with detailed information on bulge-to-disk ratio, inclination and disk size are underway, making these databases ideal for determinations of SFR using radiative transfer models. Andreas Zezas presented "The Star-Formation Reference Survey" (Ashby et al. 2011), a unique statistical sample of 369 galaxies selected to cover all types of star-forming galaxies in the nearby universe. The survey overlaps with the SDSS and NVSS areas and has GALEX, SDSS, 2MASS, Spitzer and NVSS multiband photometry and planned bulge-disk decompositions of optical images, which will make it ideal for self-consistent and systematic determinations of SFRs. It will also asses the influence of AGN fraction and environment on SFR. 
- Denis Burgarella presented applications of the CIGALE SED fitting method on the Lyman break galaxies at $2.5<z<4$ detected in the Far-infrared with Herschel and implication for star formation determinations at high redshift.

- Andrew Hopkins showed results on SFRs derived from applications of the energy balance method on the GAMA survey. Applications of radiative transfer techniques to the de-reddening of GAMA galaxies by Grootes et al. (2013) were presented in the review of C. Popescu.

\section{Acknowledgements}

PK and JPA thank the organisers for an impressive and memorable conference. BDL acknowledges financial support from the Einstein Fellowship program. AZ acknowledges financial suppor from NASA in the form of an ADAP program, and the EU IRG grant 224878. Space Astrophysics at the University of Crete is supported by EU FP7-REGPOT grant 206469 (ASTROSPACE).

\section{References}

Allen, R. J., et al. 1997, ApJ, 487, 171

Allen, L., Megeath, S. T., Gutermuth, R., et al. 2007, Protostars and Planets V, 361

Alonso-Herrero, A., et al. 2006, ApJ, 652, L83

Andersen, M., Meyer, M. R., Robberto, M., Bergeron, L. E., \& Reid, N. 2011, A\&3A, 534, A10

André, P., Men'shchikov, A., Bontemps, S., et al. 2010, A\&A, 518, L102

Ashby, M. L. N., Mahajan, S., Smith, H. A., et al. 2011, PASP 123, 1011

Baes, M., Fritz, J., Gadotti, D. A., et al. 2010, A\&A 518, L39

Banerjee, S., Kroupa, P., \& Oh, S. 2012, MNRAS, 426, 1416

Basu, S. \& Vorobyov, E. I. 2012, ApJ, 750, 30

Basu-Zych, A. R., Lehmer, B. D., Hornschemeier, A. E., et al. 2013, ApJ, 762, 45

Bianchi, S. 2008, A\&SA, 490, 461

Bigiel, F., et al. 2008, AJ, 136, 2846

Blitz, L. \& Rosolowsky, E. 2006, ApJ, 650, 933

Bochanski, J. J., Hawley, S. L., Covey, K. R., et al. 2010, AJ, 139, 2679

Boissier, S., Boselli, A., Buat, V., et al. 2004, A\&A 424, 465

Boissier, S., et al. 2007, ApJS, 173, 524

Boissier, S. 2012, "Star Formation in Galaxies", in Planets, Stars and Stellar Systems, SpringerVerlag GmbH Berlin Heidelberg, Oswalt, T. D., McLean, I. S., Bond, H. E., French, L., Kalas, P., Barstow, M. A., Gilmore, G. F., Keel, W. C. (Eds.), Volume 6, chapter 111

Boroson, B., Kim, D.-W., \& Fabbiano, G. 2011, ApJ, 729, 12

Boselli, A., et al. 2009, ApJ 706, 1527

Braine, J., et al. 2010, A\&SA, 518, L69

Brandt, W. N. \& Hasinger, G. 2005, ARAA, 43, 827

Brandt, W. N. \& Alexander, D. M. 2010, Proceedings of the National Academy of Science, 107, 7184

Braun, R. 1997, ApJ, 484, 637

Bruzual, G. \& Charlot, S. 2003, MNRAS, 344, 1000

Buat, V., Takeuchi, T. T., Iglesias-Páramo, J., et al. 2007, ApJS, 173, 404

Burgarella, D., Buat, V., \& Iglesias-Páramo, J. 2005, MNRAS 360, 1413

Calzetti, D., Kennicutt, R. C., Jr., Bianchi, L., et al. 2005, ApJ, 633, 871

Calzetti, D., Kennicutt, R. C., Engelbracht, C. W., et al. 2007, ApJ, 666, 870

Calzetti, D., Chandar, R., Lee, J. C., et al. 2010, ApJ (Letters), 719, L158

Calzetti, D., Wu, S.-Y., Hong, S., et al. 2010, ApJ, 714, 1256

Calzetti, D. 2012, Proceedings of the XXIII Canary Islands Winter School of Astrophysics: 'Secular Evolution of Galaxies', edited by J. Falcon-Barroso and J. H. Knapen 
Cappellari, M., McDermid, R. M., Alatalo, K., et al. 2012, Nature, 484, 485

Casoli, F., et al. 1998, A\& A, 331, 451

Cenarro, A. J., Gorgas, J., Vazdekis, A., Cardiel, N., \& Peletier, R. F. 2003, MNRAS, 339, L12

Chabrier, G. 2003, PASP, 115, 763

Colbert, E. J. M., et al. 2004, ApJ, 602, 231

Combes, F. 2012, Journal of Physics Conference Series, 372, 012041

Corbelli, E., et al. 2011, A\&\&A, 528, A116

Cowie, L. L., Barger, A. J., \& Hasinger, G. 2012, ApJ, 748, 50

Crowther, P. A., Schnurr, O., Hirschi, R., et al. 2010, MNRAS, 408, 731

da Cunha, E., et al. 2008, MNRAS 388, 1595

da Silva, R. L., Fumagalli, M., \& Krumholz, M. 2012, ApJ, 745, 145

Dabringhausen, J., Kroupa, P., \& Baumgardt, H. 2009, MNRAS, 394, 1529

Dabringhausen, J., Kroupa, P., Pflamm-Altenburg, J., \& Mieske, S. 2012, ApJ, 747, 72

Daddi, E., Dickinson, M., Morrison, G., et al. 2007, ApJ 670, 156

Dale, D. A. \& Helou, G. 2002, ApJ 576, 159 MNRAS 288, 1595

Dale, D. A., et al. 2009, ApJ 703, 517

Davé, R. 2008, MNRAS 385, 147

Devriendt, J. E. G., Guiderdoni, B., \& Sadat, R. 1999, A\&SA 350, 381

de Looze, I., Baes, M., Bendo, G., et al. 2012a, MNRAS 427, 2797

de Looze, I., Baes, M., Fritz, J., \& Verstappen, J. 2012b, MNRAS 419, 895

De Marchi, G., Paresce, F., \& Pulone, L. 2007, ApJ (Letters), 656, L65

de Wit, W. J., Testi, L., Palla, F., Vanzi, L., \& Zinnecker, H. 2004, A\&AA, 425, 937

de Wit, W. J., Testi, L., Palla, F., \& Zinnecker, H. 2005, A\& $A, 437,247$

Dib, S., et al. 2011, MNRAS, 415, 3439

Dijkstra, M., Gilfanov, M., Loeb, A., \& Sunyaev, R. 2012, MNRAS, 421, 213

Domínguez Sánchez, H., Mignoli, M., Pozzi, F., et al. 2012, MNRAS, 426, 330

Draine, B. T. \& Li, A. 2007, ApJ 657, 810

Driver, S. P., Hill, D. K., \& Kelvin, L. S. et al. 2011, MNRAS 413, 971

Eales, S., Dunne, L., Clements, D., et al. 2010, PASP 122, 499

Elbaz, D., Daddi, E., Le Borgne, D., et al. 2007,A $\& A$ 468, 33

Elmegreen, B. G. \& Parravano, A. 1994, ApJ (Letters), 435, L121

Engargiola, G., et al. 2003, ApJS, 149, 343

Engelbracht, C. W., Gordon, K. D., Rieke, G. H., et al. 2005, ApJ (Letters), 628, L29

Fabbiano, G. 1989, ARAA, 27, 87

Fabbiano, G. 2006, ARAA, 44, 323

Figer, D. F. 2005, Nature, 434, 192

Fragos, T., Lehmer, B., Tremmel, M., et al. 2013, ApJ, 764, 41

Fumagalli, M., et al. 2011, ApJ 741, L26

Gardan, E., et al. 2007, A\&A A73, 91

Gilfanov, M. 2004, MNRAS, 349, 146

Gratier, P., et al. 2012, A\&SA, 542, 108

Gratier, P., et al. 2010, A\&\&A, 522, 3

Grootes, M., Tuffs, R. J., Popescu, C. C., Pastrav, B. A., Andrae, E. et al. 2013, ApJ, submitted

Groves, B., Dopita, M. A., Sutherland, R. S., Kewley, L. J., Fischera, J., Leitherer, C., Brandl, B., \& van Breugel, W. 2008, ApJS 176, 438

Gvaramadze, V. V., Weidner, C., Kroupa, P., \& Pflamm-Altenburg, J. 2012, MNRAS, 424, 3037

Heavens, A., et al. 2004, Nature, 428, 625

Hennebelle, P. 2012, A\&A A, 545, A147

Hopkins, A. M. 2004, ApJ, 615, 209

Hao, C.-N., et al. 2011, ApJ 741, 124

Hirashita, H., et al. 2003, A\&A 410, 83

Hunter, D. A., et al. 1986, ApJ, 303, 171

Hsu, W.-H., Hartmann, L., Allen, L., et al. 2012, ApJ, 752, 59

Iglesias-Páramo, J., Buat, V., Takeuchi, T. T., et al. 2006, ApJS, 164, 38 
Iwasawa, K., et al. 2009, ApJ (Letters), 695, L103

Kennicutt, R. C. $1989, A p J, 344,685$

Kennicutt, R. C. 1998, ARAA 36, 189

Kennicutt, R. C., et al. 2009, ApJ 703, 1672

Kennicutt, R. C. \& Evans, N. J. 2012, ARAA 50, 531

Koen, C. 2006, MNRAS, 365, 590

Köppen, J., Weidner, C., \& Kroupa, P. 2007, MNRAS, 375, 673

Kroupa, P. 2001, MNRAS, 322, 231

Kroupa, P. 2002, Science, 295, 82

Kroupa, P., Aarseth, S., \& Hurley, J. 2001, MNRAS, 321, 699

Kroupa, P. \& Bouvier, J. 2003a, MNRAS, 346, 343

Kroupa, P. \& Bouvier, J. 2003b, MNRAS, 346, 369

Kroupa, P., Bouvier, J., Duchêne, G., \& Moraux, E. 2003, MNRAS, 346, 354

Kroupa, P. \& Gilmore, G. F. 1994, MNRAS, 269, 655

Kroupa, P. \& Weidner, C. 2003, ApJ, 598, 1076

Kroupa, P., Weidner, C., Pflamm-Altenburg, J., et al. 2013, in Planets, Stars and Stellar Systems, Vol 5.: Stellar Systems and Galactic Structure, Gilmore, G. (Ed.), Springer (ArXiv:astro$\mathrm{ph} / 1112.3340)$

Kylafis, N. D. \& Misiriotis, A. 2006, in "The many scales in the Universe", JENAM 2004 Astrophysics Reviews, eds. J. C. Del Toro Iniesta, E. J. Alfaro, J. G. Gorgas, E. SalvadorSole, \& H. Butcher, p. 111

Lada, C. J. \& Lada, E. A. 2003, ARA\&A, 41, 57

Laird, E. S., Nandra, K., Hobbs, A., \& Steidel, C. C. 2006, MNRAS, 373, 217

Lamb, J. B., Oey, M. S., Werk, J. K., \& Ingleby, L. D. 2010, ApJ, 725, 1886

Lee, J. C., et al. 2009, ApJ 706, 599

Lehnert, M. D. \& Heckman, T. M. 1996, ApJ, 472, 546

Lehmer, B. D., et al. 2008, ApJ, 681, 1163

Lehmer, B. D., et al. 2010, ApJ, 724, 559

Leroy, A., Bolatto, A., Walter, F., \& Blitz, L. 2006, ApJ, 643, 825

Leroy, A. K., et al. 2008, AJ 136, 2782

Li, H.-N., et al. 2007, AJ, 134, 1315

Li, Y., Crocker, A. F., Calzetti, D., et al. 2013, ApJ, 768, 180

Linden, T., Kalogera, V., Sepinsky, J. F., et al. 2010, ApJ, 725, 1984

Liu, G., et al. 2011, ApJ 735, 63

MacLachlan, J. M., Matthews, L. D., Wood, K., \& Gallagher, J. S. 2011, ApJ 741, 6

Marks, M. \& Kroupa, P. 2012, A\& A, 543, A8

Marks, M., Kroupa, P., Dabringhausen, J., \& Pawlowski, M. S. 2012, MNRAS, 422, 2246

Marshall, J. A., Herter, T. L., Armus, L., Charmandaris, V., Spoon, H. W. W., Bernard-Salas, J., \& Houck, J. R. 2007, ApJ 670, 129

Martin, C. L. \& Kennicutt, Jr., R. C. 2001, ApJ, 555, 301

Maschberger, T. \& Clarke, C. J. 2008, MNRAS, 391, 711

Maschberger, T., Clarke, C. J., Bonnell, I. A., \& Kroupa, P. 2010, MNRAS, 404, 1061

Meurer, G. R., et al. 1995, AJ 110, 2665

Meurer, G. R., et al. 2009, ApJ 695, 765

Mineo, S., Gilfanov, M., \& Sunyaev, R. 2012a, MNRAS, 419, 2095

Mineo, S., Gilfanov, M., \& Sunyaev, R. 2012b, MNRAS, 426, 1870

Mineo, S., Gilfanov, M., Lehmer, B. D., Morrison, G. E., \& Sunyaev, R. 2014, MNRAS, 437, 1698

Misiriotis, A., Popescu, C. C., Tuffs, R. J., \& Kylafis, N. D. 2001, A\& A, 372, 775

Molinari, S., et al. 2010, A\&A, 518, L100

Moore, C. A., et al. 2010, AJ 140, 253

Murgia, M., et al. 2002, A\&SA, 385, 412

Natale, G., Tuffs, R. J., Xu, C. K., Popescu, C. C., \& Fischera, J. et al. 2010, ApJ 725, 955

Neininger, N., et al. 1998, Nature, 395, 871 
Nieten, C., et al. 2006, A\& A, 453, 459

Noll, S., et al. 2009, A\&\&A 507, 1793

Norman, C., Ptak, A., Hornschemeier, A., et al. 2004, ApJ, 607, 721

Oosterloo, T., et al. 2007, AJ, 134, 1019

O'Sullivan, E., Forbes, D. A., \& Ponman, T. J. 2001, MNRAS, 328, 461

Oey, M. S. \& Clarke, C. J. 2005, ApJ (Letters), 620, L43

Oh, S. \& Kroupa, P. 2012, MNRAS, 424, 65

Padoan, P. \& Nordlund, Å. 2002, ApJ, 576, 870

Padoan, P., Nordlund, Å., Kritsuk, A. G., Norman, M. L., \& Li, P. S. 2007, ApJ, 661, 972

Pastrav, B. A., Popescu, C. C., Tuffs, R. J., \& Sansom, A. E. 2013, A\&GA, 557, A137

Pereira-Santaella, M., et al. 2011, A\&SA, 535, A93

Persic, M. \& Rephaeli, Y. 2007, A\&A A, 463, 481

Pflamm-Altenburg, J. \& Kroupa, P. 2009, ApJ, 706, 516

Pflamm-Altenburg, J. \& Kroupa, P. 2010, MNRAS, 404, 1564

Pflamm-Altenburg, J., Weidner, C., \& Kroupa, P. 2007, ApJ, 671, 1550

Pflamm-Altenburg, J., Weidner, C., \& Kroupa, P. 2009, MNRAS, 395, 394

Popescu, C. C., Misiriotis, A., Kylafis, N. D., Tuffs, R. J., \& Fischera, J., 2000, A $\mho A$ 362, 138

Popescu, C. C., Tuffs, R. J., Kylafis, N. D., \& Madore, B. F. 2004, A 6 A 414, 45

Popescu, C. C., Tuffs, R. J., Madore, B. F., et al. 2005, ApJ 619, L75

Popescu, C., et al. 2011, A\&\&A 527, 109

Prescott, K. M., et al. 2007, ApJ 668, 182

Ptak, A., et al. 2007, ApJ, 667, 826

Ranalli, P., Comastri, A., \& Setti, G. 2003, A\&A A, 399, 39

Ranalli, P., Comastri, A., Zamorani, G., et al. 2012, A\&A, 542, A16

Recchi, S., Calura, F., \& Kroupa, P. 2009, A\&A, 499, 711

Reddy, N. A. \& Steidel, C. C. 2004, ApJ (Letters), 603, L13

Reggiani, M. M. \& Meyer, M. R. 2011, ApJ, 738, 60

Relaño, M., Kennicutt, Jr., R. C., Eldridge, J. J., Lee, J. C., \& Verley, S. 2012, MNRAS, 423, 2933

Rosolowsky, E. 2005, PASP, 117, 1403

Rosolowsky, E., et al. 2008, A\&AR, 15, 189

Sajina, A., Scott, D., Dennefeld, M., Dole, H., Lacy, M., \& Lagache, G. 2006, MNRAS 369, 939

Schaerer, D. \& de Barros, S. 2013, A\& A 549, 4

Schaye, J. 2004, ApJ, 609, 667

Schechtman-Rook, A., Bershady, M. A., \& Wood, K. 2012, ApJ 746, 70

Schilbach, E. \& Röser, S. 2008, A\&A, 489, 105

Schuller, F., et al. 2009, A\&A, 504, 415

Sharma, S., Corbelli, E., Giovanardi, C., Hunt, L. K., \& Palla, F. 2011, A\&̋A, 534, A96

Siebenmorgen, R., Krügel, E., \& Spoon, H. W. W. 2004, A\&A A, 414, 123

Siebenmorgen, R. \& Krügel, E. 2007 A $\mathscr{J} A, 461,445$

Silva, L., et al. 1998, ApJ 509, 103

Stamatellos, D., Hubber, D. A., \& Whitworth, A. P. 2007, MNRAS, 382, L30

Symeonidis, M., Georgakakis, A., Seymour, N., et al. 2011, MNRAS, 417, 2239

Testi, L., Palla, F., \& Natta, A. 1998, A\&A, 133, 81

Testi, L., Palla, F., Prusti, T., Natta, A., \& Maltagliati, S. 1997, A\&A, 320, 159

Thies, I. \& Kroupa, P. 2007, ApJ, 671, 767

Thies, I. \& Kroupa, P. 2008, MNRAS, 390, 1200

Thies, I., Kroupa, P., Goodwin, S. P., Stamatellos, D., \& Whitworth, A. P. 2010, ApJ, 717, 577

Tremonti, C. A., Heckman, T. M., Kauffmann, G., et al. 2004, ApJ, 613, 898

Toomre, A. 1964, ApJ, 139, 1217

Tuffs, R. J., Popescu, C. C., Völk, H. J., Kylafis, N. D., \& Dopita, M. A. 2004, A $6 A$ 419, 821

Tzanavaris, P. \& Georgantopoulos, I. 2008, A\&A A, 480, 663

van Dokkum, P. G. \& Conroy, C. 2010, Nature, 468, 940

van Dokkum, P. G. \& Conroy, C. 2011, ApJ (Letters), 735, L13 
Vattakunnel, S., Tozzi, P., Matteucci, F., et al. 2012, MNRAS, 420, 2190

Walcher, J., et al. 2010, ApESSS 331, 1

Weidner, C. \& Kroupa, P. 2004, MNRAS, 348, 187

Weidner, C. \& Kroupa, P. 2005, ApJ, 625, 754

Weidner, C. \& Kroupa, P. 2006, MNRAS, 365, 1333

Weidner, C., Kroupa, P., \& Bonnell, I. A. D. 2010, MNRAS, 401, 275

Weidner, C., Kroupa, P., \& Larsen, S. S. 2004, MNRAS, 350, 1503

Weidner, C., et al. 2011, MNRAS, 412, 979

Weisz, D. R., Johnson, B. D., Johnson, L. C., et al. 2012, ApJ, 744, 44

Whitworth, A., Bate, M. R., Nordlund, Å., Reipurth, B., \& Zinnecker, H. 2007, Protostars and Planets V, 459

Whitworth, A., Stamatellos, D., Walch, S., et al. 2010, in IAU Symposium, Vol. 266, IAU Symposium, ed. R. de Grijs \& J. R. D. Lépine, 264-271

Whitworth, A. P., Bhattal, A. S., Chapman, S. J., Disney, M. J., \& Turner, J. A. 1994, MNRAS, 268,291

Wilkins, S. M., et al. 2008, MNRAS, 385, 687

Wilson, C. D. 1997, ApJ (Letters), 487, L49

Wu, H., Cao, C., Hao, C.-N., et al. 2005, ApJ (Letters), 632, L79

Wu, H., Zhu, Y.-N., Cao, C., \& Qin, B. 2007, ApJ, 668, 87

Wuyts, S., et al. 2011, ApJ 738, 106

Xu, C., Hacking, P. B., Fang, F., Shupe, D. L., Lonsdale, C. J., Lu, N. Y., Helou, G., Stacey, G. J., \& Ashby, M. L. N.. 1998, ApJ 508, 576

Young, J. S. \& Knezek, P. M. 1989, ApJ (Letters), 347, L55

Yuan, F.-T., et al. 2011, PASJ, 63, 1207

Zhu, Y.-N., et al. 2008, ApJ 686, 155 